\title{
RAPID ATHLETE TESTING
}

\author{
BY \\ MARK EDWARD FOX
}

A thesis submitted to the Victoria University of Wellington

in fulfilment of the requirements for the degree of

Masters of Innovation and Commercialisation.

Victoria University of Wellington

2017 


\section{TABLE OF CONTENTS}

RAPID ATHLETE TESTING




\section{Abstract}

The Rapid Athlete Testing project stemmed from exploratory research partnership to assess the commercial viability of Auramer Bio's aptamer-based biosensor in the field of testing athletes for signs of drug abuse and doping in sport. Within this project, we aimed to explore a side avenue for the device to ascertain further market exploration and to determine whether it could be a popular and profitable industry to enter.

The drug abuse and doping in sport industry is a worldwide cause and the World AntiDoping Agency (WADA) leads the charge as an individual governing body. Each nation has a government-affiliated body that facilitates the sample collecting and testing at one of the 34 WADA accredited labs around the world. The scope of the opportunity for the innovation and commercialisation of this project is immense as Auramer Bio's device could be implemented on the grand worldwide scale.

This project and report aims to explore and analyse the development and commercialisation potential of the device for Auramer Bio. The opportunity primarily lay in the evaluation of a suitable business and product development strategy, understanding the scope of the industry and a thorough analysis of similar technologies and competitor products.

In order to delve into this regimented and secretive industry to extract the necessary information and assess the viability of our goal, we first needed to find where we could obtain these sources of information and what we needed to ask them. For the true development of the project, we explored several business and development strategies to ensure the right methodology was being carried out for the device. We assessed our most direct competition and investigated the development strategies of similar technologies. Next, we began to reach out and connect with organisations involved with the drug testing in sport industry, as well as those indirectly involved and those affected by it. We directly interviewed candidates from Drugfree Sport NZ, the Australian Sports Drug Testing Lab (ASDTL) and were even fortunate enough to have a survey for WADA answered.

Through our investigation, we found many key inefficiencies within the industry that our device could exploit to create a viable niche. Our research participants confirmed our base 
theories and assumptions, whilst also delivering vital information that allowed us to pivot accordingly. We have also found an amiable starting point for the device's prototype and a direction for the future. With the help of one of our research participants, we were able to isolate a plan for our path to market and our hopeful future WADA accreditation for the device.

The Rapid Athlete Testing project has sought out the knowledge of several key industry representatives, the athletes and coaches themselves and has networked effectively to create a practical starting point for Auramer Bio's aptamer based biosensor within a firmly structured industry. If managed successfully in the future, the work from this project could take the device to the world stage and be a key tool in the Olympics and other large sporting events to come, maintaining fair competition in sport. 
First and foremost, I'd like to acknowledge and thank Jenny Douché. Without her knowledge, help and patience, this project would never have come to fruition.

Secondly, I'd like to thank Jeremy Jones and Justin Hodgkiss from Auramer Bio, for being great mentors, for helping supervise the project and for the opportunity to act as an intrapreneur and explore a market l'd never even considered. You two have greatly broadened my horizons.

Lastly, I'd like to thank my family for their continued support as I trickle through another year at University. 


\section{Foreword:}

For this report, I use the term "Athlete" to describe any individual that participates in highlevel sport, not just running or marathon-style events. It is in my opinion, the perfect gender-neutral term to describe anyone that fits into this description and is largely preferential to the term "sports-person".

I also refer to myself within the report as the "lead researcher", as that was my role within the project in partnership with Auramer Bio. This was to avoid using "I" phrases and maintain the tone and tense of the report. 


\section{Introduction:}

The world of sport is fundamentally dynamic, in tandem with the world of drug abuse and doping in sport. The earliest recollections of performance enhancement stem from the Ancient Olympics, where athletes ate figs in an attempt to improve performance (Holt et al, 2009). With the historically recent rise in understanding of science, chemistry and pharmacology, athletes have been reported to experiment with many different mixtures of drugs to overcome their competition. From the abuse of Methamphetamine to Human Growth Hormone, several deaths occurred and the demand for the development of a policy to ban performance enhancers in sport reached its peak. The World Anti-Doping Agency (WADA) was founded in 1999 as an independent, international agency funded by the sport movement and government agencies of the world. WADA are dedicated to education, scientific research, development of anti-doping capacities and supervising the World AntiDoping Code.

The pressure put on athletes to succeed are phenomenal, to the point where the outcome of a big event can determine whether the athlete can receive scholarships, sponsorship or be able to compete in subsequent events. Anti-doping laws, policies and testing techniques have had to follow suit and be just as vigorous. The World Anti-Doping Association is the global body that governs the fight against drug abuse and doping in sport. Each country has its own government constituent anti-doping body that works with the athletes, collects the samples, organises the tests and then delves into the legal aspect to prosecute any infringements. All athlete's samples, whether they are blood or urine, are collected by sample collectors of the country's anti-doping body. These samples are placed into an " $A$ " and " $\mathrm{B}$ " bottle, sealed with tamper-proof tape, packaged into a tamper-proof kit and sent to the closest of the 34 WADA accredited labs around the globe. Depending on what is being tested, the size of the event and whether the athlete is in their on or off season, the results can take up to four weeks. This procedure, although developed to be secure, appears quite cumbersome and could be made a lot more efficient. 
This project has been named Rapid Athlete Testing and aims to explore the anti-doping in sport industry, understand its current policies, procedures, legislation and to network and converse with relevant individuals within the industry. This project also aims to examine the prospect and commercial viability that Auramer Bio's aptamer-based biosensor could be a useful tool within the anti-doping framework by adding efficiency alongside the potential for a large financial saving.

\section{The Opportunity}

The Rapid Athlete testing project started as a birthchild of the Masters of Innovation and Commercialisation programme run by Victoria University of Wellington. This started as a partnership for the researcher to act as an intrapreneur or subsidiary company for Auramer Bio. Auramer Bio are a Biotechnology company that generates aptamers for commercial use. Aptamers are single-stranded deoxyribonucleic acid (DNA) or ribonucleic acid (RNA) that will only bind to a complementary target strand, making them highly specific. Auramer Bio has under development a portable, handheld, aptamer-based biosensor that can detect highly specific targets. The current industry detection standard operates on the Pico level $\left(10^{-12)}\right.$ Auramer Bio's potential device can detect targets 1000 times more accurately down to the Femto level $\left(10^{-15}\right)$.

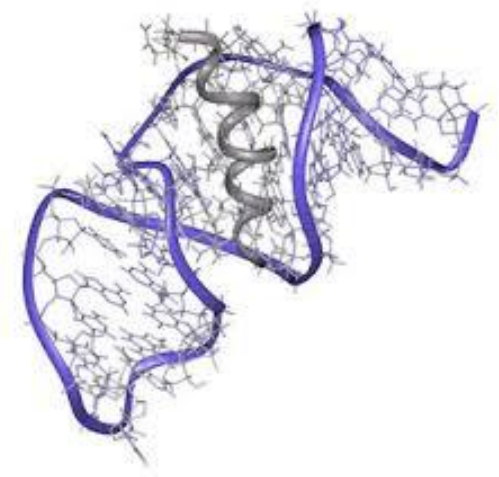

Figure 1: Example of Aptamer Binding to Target, Aptamer Diagnostic Biochips, 2017.

The end goal is to be able to detect a target and produce a result within a reasonable timeframe of approximately 20 minutes. Their main avenue of exploration had been to utilise this biosensor to differentiate levels of hormones that reveal whether a woman is incredibly stressed, or undergoing early menopause as the current methods involve several tests that are required to tell the difference. Initially, we wanted to explore the possibility to 
detect early signs of gynecomastia in pre-pubescent boys, however the current medical research had not presented a clear target for an indication of the hormonal irregularity. The next area of interest then lay in testing athletes for signs of drug abuse and doping in sport, which lay the foundation for the project to commence.

After investigating the current sample collection and testing methods, the opportunities for the development of the project were vast and the potential scope for the project was very overwhelming. If the device itself could gain the appropriate WADA accreditation and be shown to work to a high and consistent standard, it could be put in the sample collector's hands and circumvent the lab stage entirely. It could also be used in the labs as this is more secure and would create a large saving for the labs themselves, especially on the more expensive lab tests, creating a large incentive to utilise this technology. Finally, there is also the opportunity for it to be used as an onsite, confidential service for coaches, athletes and teams that wish to do an internal investigation without the blowback and embarrassment from the media.

\section{The Business Model and Development Strategy}

The proposed development strategy for the true growth of this project and the device, which is still in its lab component state and not a prototype, will be to follow the Waterfall method to discover the requirements necessary to fulfil the legislative need imposed by the various government derivatives of WADA. We will then work on design and implementation to develop a minimal viable product. The Testing phase is the next stage to prove that the device works and can pass all the protocols required for accreditation, this will require a lot of peer reviewed studies. The device is then ready for the release and maintenance stage where we can actively sell the device along with the required aptamer kits. Following the initial Waterfall, the lean method can then be utilised to develop, produce and release the required aptamer kits to detect new drugs as they become prevalent or are added to the WADA prohibited list. 

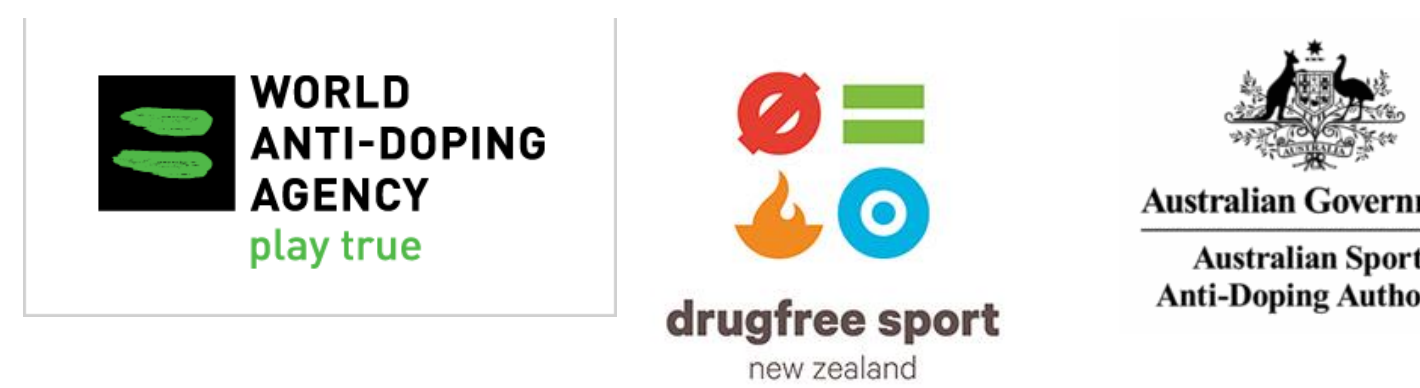

Australian Government

Australian Sports Anti-Doping Authority

Figure 2: Anti-doping agency emblems

The business model for this project couldn't have been developed without first understanding the customer segment and acquiring a list of all the potential stakeholders involved. To better understand and illustrate this, we developed a business model canvas (see Appendix A). By understanding the current sample collecting and testing methods, we have been able to see where the biosensor can be utilised by the customer segment, the value propositions that we can present, our key partners and activities, the key resources required, the delivery channels and an outline of the revenue streams and cost structures for the future of the project. The business model canvas highlights and accentuates the scope for the starting point of Rapid Athlete Testing, bolstering the scope of the project even at its starting point in New Zealand and Australia. The main objectives of this project have been to explore the market feasibility of Auramer Bio's aptamer-based biosensor within the anti-doping and drug abuse in sport industry. We aim to identify whether there is a market need for a portable, handheld aptamer-based biosensor that can detect highly specific targets and render a result within a reasonable timeframe. Another objective is to network effectively with potential stakeholders to build strong business relationships which are essential for any start up.

\section{Technology and Competitor Analysis}

There has been a lot of related research in the field of drug testing for athletes using new and innovative testing methods. Affinity-based biosensors have received a lot of commendations as they are cheap, easy to use and selective analysis devices, characterised with a reversible reaction with the analyte being investigated, allowing for multiple analyses using the same sensor (Mazzei et al, 2014). However, as they are so specific there would 
need to be a constant stream of new analyte detectors as more drugs get added to the WADA prohibited list and as more synthetic anabolic steroids are produced. According to WADA statistics, androgenic anabolic steroids (AAS) are the most preferred doping method for athletes since the current WADA mass spectrometry identification criteria cannot be applied to unknown molecules (Abushareeda et al, 2014). This means that by using these designer AAS, athletes can potentially achieve the desired performance enhancement, whilst not being caught through the WADA tests. Another main avenue of related research for this project has lay within competitor's and their products. The most direct competition would be a device stemming out of Tomsk Polytechnic University in Russia, where they have developed an electrochemical sensor capable of detecting a desired target and producing the result on the user interface screen very quickly. Currently this device only tests for Meldonium, a drug that was put on the WADA prohibited list in May 2016, Meldonium is commonly prescribed for treating high blood pressure. Athletes have been using Meldonium for its properties of opening blood vessels to help deliver nutrients to the muscles and organs more quickly for faster recovery.

The development and commercialisation activities of affinity based biosensors and electrochemical sensors for the use of testing athletes for signs of drug abuse and doping in sport may vary slightly depending what they are detecting. There must clearly be a significant amount of evidence to show that these devices are fit for the purpose and would require multiple peer reviews to be considered, let alone accepted as these innovators would not want their devices to produce false positives or false negative results. These devices could effectively change a global system and practice of testing athletes for signs of drug abuse an doping in sport, so in order to be integrated into the sampling or testing methodologies, there function, operation and delivery of results must be absolute. Overall, the use of these devices to test athlete's samples comes down to WADA's decision to work with these devices and their companies, their effectiveness and their cost. After considering all of these factors WADA may integrate their use into the already established routines. 


\section{Literature Review for Industry Analysis}

The Lean Startup: How Today's Entrepreneurs Use Continuous Innovation to Create Radically Successful Business by Eric Ries was the first literature insight into business model and strategy that we explored. The lean method embodies a notion that the product, service or innovation in mind can have some base research but is released as a "minimal viable product", that covers all the basic functions. From there, through customer and user feedback, updates or iterations are released to include more utilities and will continue to develop as more feedback is provided. Our device could be released, and through the feedback of our stakeholders and customers we could continuously improve it. At first, this appeared to be a sound strategy for the project, but after some investigation and the realisation that we absolutely did not want to produce false positives or negatives for fear of being sued, this particular model was decided to be ineffective for the niche market we are endeavouring to enter.

The Startup Owner's Manual: The Step-by-Step Guide for Building a Great Company by Steve Blank and Bob Dorf provided a plethora of knowledge on customer selection, business development and business model strategies. This literature aided in the decision-making process and how to conduct ourselves as we investigated different strategies to implement to further the development of the project.

If You Build It, Will They Come? Three Steps to Testing and Validate Any market Opportunity by Rob Adams gifted great market research advice whilst defining the jargon we might run into whilst conducting our research. This literature outlines a proven and effective means for market validation and testing strategy, how to assess the direct and non-direct competitors and their products. There are tips on interview strategy, determining what the market really wants and how to include the feedback on what customers say they want. This literature also brought up the very valid point that we needed to consider: technology is forever advancing. This meant we needed to look into our business strategy and realise that this device may have a finite life on the market before something more efficient, costeffective and accurate becomes viable and supersedes our device, this is a broad market trend that we have no control over, yet must still consider. Adams also promotes a checklist to evaluate whether the proposed product is ready for release to the market: this includes domain knowledge, market knowledge, understanding life cycles and trends of similar 
technology, analysing competitors and their products, economic analysis and reviewing what the experts are saying. This literature has been essential to not only the business strategy and the project development, but to the skills of the lead researcher to better gauge the interest of the stakeholder and customer base and how to truly discover their needs.

Business Models, Business Strategy and Innovation by David J. Teece aided in the formation of the development strategy and business model. Teece explains that the modern innovation sector and start-up companies are now more focused on a "customer-centric" model and strategy. This is due to the rapid evolution of technology, often giving customers and stakeholders a great deal of information and potential solutions. Based on this literature, it was decided that we would need to drastically limit our assumptions and learn what was relevant to truly address the customers' needs and how to capture the value of these. Our device could then be made to specification and be completely ready to enter and actively compete within our desired market.

\section{Project Management Methodologies: Selecting, Implementing and Supporting}

Methodologies and Processes for Projects by Jason Charvat, provided background knowledge or the Waterfall method (see Appendix B). Waterfall started in the 1970's as an effective model at the time for software development, however it has had several revisions since then and many forms of the waterfall method have arisen because of it. Charvat describes the benefits and disadvantages of the Waterfall method for us to consider, as it provides a regimented structure, a clear indication of the next stage and an iterative approach. However, there is also minimal feedback between phases, results of the project only occur later in the life cycle and each stage may be restricted by time.

For our project, the device is still in its lab component stage and not ready to be made into a prototype. Also, given the legislation and policies for the sample collecting and lab testing processes easily accessible on the WADA website, the waterfall development strategy and business model was deemed most appropriate for the project. Through this development strategy, we can discover the requirements and needs of the target customers and stakeholders. We can then work on design and implementation to lift the lab components into a prototype minimal viable product. Following this, suitable testing and peer revision will be required to prove that the device works and can pass all the protocols required for 
proper accreditation. The device is then moved into the final release and maintenance stage where our customers can use the device. After the release and maintenance stage, the lean method (Ries, 2011) can be utilised to develop, produce and release the required aptamer kits to detect new drugs as they become prevalent or as new drugs are added to the prohibited list.

\section{Theories about the Project}

The main theories that helped guide the projects development relied on the idea that this was a novel solution to the current perceived customer problem and that this device could add a critical element of efficiency to a system that appears to lack it. We also believed that as this device is more accurate than the current industry it could be utilised to be more scrupulous in the testing field when it comes to detecting trace elements or remnants of a target. The other main theory is that there is always demand to make a system more efficient and create a saving for stakeholders, so therefore this project could be disruptive, time and cost efficient as well as commercially viable.

Further investigation is needed primarily in the requirements section of the project development model, as we will need to know all the required protocols to pass to be able to develop a prototype that fits the need of the stakeholder and the market. These rigorous protocols must be met because if the device accidentally produces a false result, then serious legal ramifications may ensue, which could ultimately lead to the device being excluded from the WADA process and we may lose a potential market niche. Further investigation also lies in the development area, as the device is currently still in its lab component state, we need to assess how long it will take to develop a minimal viable product after creating a working prototype. Whilst background research is possible to understand the fundamentals of the WADA process, the real starting point for the device to reach the testing stage of the development plan is currently limited by the development of the device. Once a minimal viable product is reached, there will need to be many tests and peer reviewed investigations to prove the device works and can be considered to enter this meticulous market.

The key assumptions about the project is that we assume we are developing a device that our proposed customer base will want and that we can make and sell the device and its 
aptamer kits profitably. We also assume that there is a legitimate need for our device and that there is a sufficient customer base. These assumptions are to be verified through extensive market research and following our business plan. Finally, we assume the project will become a profitable venture, be managed effectively in terms of financial and legal implications and create adequate capitalisation to pay off the initial start-up costs as well as research and development. The business plan will be generated around managing these assumptions to ensure preliminary and future success.

\section{Project Scope, Requirements and Limitations}

The project's scope is part of the planning process and involves a specific list of goals, deliverables, features, functions, tasks, deadlines and cost. For the Rapid Athlete Testing project, the scope involved defining the requirements of the device, the process requirements for our customer base, involvement of the correct stakeholders, identification of limitations and the overall project management.

The requirements of the device were already somewhat clear given the identified market opportunity. It had to be accurate, be able to test for multiple targets in a single run, be able to test across multiple matrices (ie: blood, urine, saliva etc.) be simple to use with a clear interface, produce the results within a reasonable timeframe and meet the legislative accreditation requirement imposed by WADA.

The process requirements for the device define how our customers will interact with us and how this device will interact existing business processes. Once the device has passed the necessary requirements to be introduced to this market, our clientele will be able to order the devices and the required aptamer kits to run the device. A catalogue of aptamers is to be produced so that the customer base can order kits to detect their desired targets, customer accounts will need to be created to accommodate the ordering, invoicing and delivery portion of the project.

Involvement of the correct stakeholders is an integral asset to the development of the project. Gauging athlete's opinions on the current methods has given great insight into how it is perceived and where there is opportunity to make things better for both parties of the sample collection process. As the project is basing its starting point in New Zealand, working alongside Drugfree Sport NZ and the Australian Sport Drug Testing Lab (ASDTL) will not only 
provide significant feedback and information for the device, but also offers a link to WADA. This will provide a strong foothold to potentially have the device integrated into the sample collecting and testing protocols not only in Oceania, but throughout all the accredited labs and sample collection agencies worldwide.

The limitations of the project stem from Auramer Bio being a start-up and that the device isn't ready to be released to the market. Currently this means that a lot of work is required to get the device to the correct standard before we can get it tested and peer reviewed. Time is the limitation as well, during the market research portion of the project many networking connections were forged and interest gauged by stakeholders and investors, should the development take too long, these connections may dwindle.

As this project is the birthchild of a Master's program, the lead researcher must hand the management of the future development of the project over to Auramer Bio. This means that they will be required to familiarise themselves with the market research, introduce themselves to the stakeholders and carry on forging plans for the project in the future. Conversely, it will be up to Auramer Bio as to whether they wish to pursue this avenue for their device, they may decide to pause their interest in this market or scrap the project altogether.

\section{Overview of the Report Structure}

Throughout the rest of this report we will explore the Methodology employed for the project, examine and discuss the Findings and analysis of the results, the Discussion of any major findings and their implications, the Recommendations or business case section is to consolidate the findings of the research and lastly there is the Conclusion chapter to summarise the key outtakes of the research. Following these will be the References and Appendices. 


\section{Methodology:}

The Rapid Athlete Testing Project was the birthchild of the partnership between Auramer Bio and the Masters of Innovation and Commercialisation course run by Victoria University of Wellington. Before this project commenced, the lead researcher pursued a partnership with Metservice to explore the use of API technology to link weather data to a personalised weather planner app. This app had the intention of knowing the user, their schedule and being able to indicate suitable times when appropriate weather was prevalent for their favourite activities. There was also the possibility to integrate a social interest formula into the app so that people interested in similar activities could meet up. However, after 3 months of exploration it was found that due to many similar products on the market and general disinterest from stakeholders and the surveyed customer base, the project was found to be inviable and was axed.

While exploring the Rapid Athlete Testing project, several assumptions were made in the development strategy and business model. We assumed that because we could deliver a test result for a substance within a reasonable timeframe of approximately 20 minutes, as opposed to the four-week turnaround in the current method, that of course our idea would be hugely appreciated, profitable and successful. We assumed that by adding a cost saving and making their system more efficient, that our product would be taken with high praise and integrated quickly into their processes to save time and money. We assumed that there is a legitimate need for our product and that our customer base is always looking for new, more efficient means to improve their sample collecting and testing methods. We also assumed that our product would fill a need within this niche that our customer potentially hadn't realised they had. Essentially, at the starting point we assumed our product bolstered efficiency, cost effectiveness and an accuracy that this market did not have in place, which would make an extremely successful avenue for the exploration and marketing of this device. These assumptions were recognised as assumptions and calibrated into the development strategy and business model. 


\section{Development Strategy and Business Model:}

We acknowledged that drug testing in sport is a heavily regimented industry, likely to have multiple facets and regulations, which we would need to discover to bring our device into this market. We isolated all of the key industry stakeholders and partners and drew them up on a Strategyzer business model canvas (see Appendix A) to contact and potentially validate our assumptions. Our business model canvas allowed us to plan and visualise our strategies in terms of our who is involved in our customer segment, how we might forge and build our customer relationships, our methods for our delivery channels as well as the value propositions that our device could potentially bring to this industry. The Strategyzer business model canvas also allowed us to discuss and highlight the key activities the device must be able to perform in order to stand out and be competitive in this market. We were then able to develop and draw up our key resources to visualise the minimum requirements our device needs and what resources may be necessary in the future for the true development of the project. Lastly, we looked at our cost structure and revenue streams to analyse where our biggest costs would come into the project to allow for initial development and start up costs, as well as future maintenance and develop strategy. Our revenue streams highlighted who from our customer segment would potentially pay for the device and the required aptamer assay kits to run the onsite tests, allowing us to plan exactly when and where money would come back to the project after the costly development phase and how our revenue stream could operate in the future.

After a lengthy literature review to identify our best development strategy, we realised that this industry isn't as fast moving as the technology or software industry, so the lean method would be detrimental. For example, if we were to release a minimum viable product and then work to fix out the bugs as it is being used by the customer base, it would make for a very costly project that, if enough issues arose, the device could be dropped by WADA as one of their biggest prerequisites is accuracy in testing. We therefore decided Waterfall was the ideal choice (see Appendix B ) as it gave us time to uncover the necessary requirements to enter this market, whilst continuing to develop from the lab component technology into a working viable prototype. As the device is still in its lab component stage and not a commercially ready prototype, we have the ability to run the device against the VRIO 
framework to ensure that our device will have the features to maintain a sustained competitive advantage within this industry and other markets.

\section{VRIO framework}

Is the Resource or Capability.

and is the Firm...

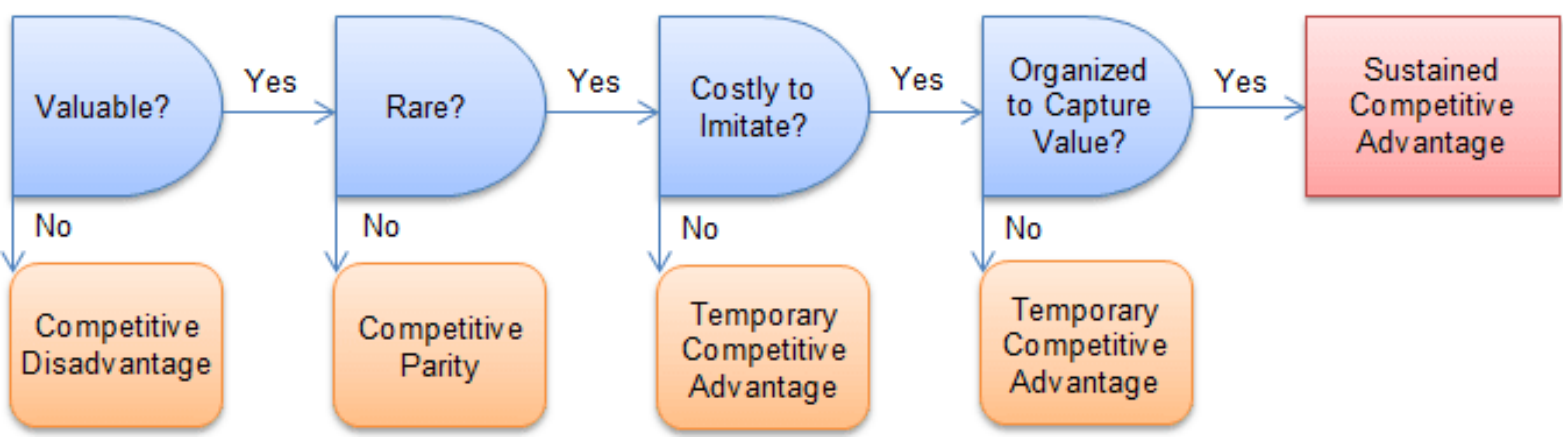

Figure 3: VRIO Framework - Strategic Management Test, 2017.

In order to collect and analyse the data, a spreadsheet of potential stakeholders was drawn up with contact name, phone number, email, the date of first contact and the date of response. As a start-up, "cold-emailing" (see Appendix C) can be a necessary and effective means to speak with relevant individuals in the industry to obtain information. We "coldemailed" WADA, Drugfree Sport NZ, the Australian Sports Anti-Doping Authority (ASADA), the Australian Sports Drug Testing Lab (ASDTL), The Drug Testing Company (TDTC) as well as several sports teams, athletes and coaches. We created specific interview questions for each potential stakeholder group to gauge their current behaviours, any barriers to changing these behaviours and their perception of the proposed product/solution (see Appendix D). We used the insight from these interviews to aid the development of the project strategy. In the case of the coaches and sports teams, we developed a generic survey to gauge their thoughts and feelings on the current testing methods and what their perception of our proposed device could bring to the process from their end (see Appendix E). We effectively culminated all of this feedback, looked for common themes amongst the athletes to present to the sports authorities and include these as features in the device to aid in the development and testing portion of the development strategy.

\section{Research Design Process:}

To develop the research design process, we looked at all of the different components of the project so that we could find ways to source information and integrate them in a coherent 
and logical manner in order to effectively address the research problem. We began with an exploratory style of research to determine all of the involved parties and wrote these on the business model canvas. We then pursued descriptive research by investigating literature, competition and examples of the commercialisation of other affinity-based biosensors, this was so we had a "rough guide" to follow for the commercialisation of our project. We then moved into explanatory research, where we attempted to connect ideas and understand the cause and effect in order to successfully understand our target market and effectively isolate the participants for research.

Firstly, we recognised that WADA was at the top of the chain and were the only organisation capable of distributing the necessary accreditation to enter the market, so it was imperative that we interview them. Secondly, as we have the assumption that our device could be used by the sample collectors and in the lab, it became apparent that we would need to speak to ASDTL, ASADA and Drugfree Sport NZ to gauge their thoughts on our device, what extra features would be beneficial and whether they thought it could be integrated effectively into their already-established chain. We spoke to coaches and athletes for their thoughts on the current processes as well. Lastly, our research plan included an aspect of nonexperimental research as we relied on the interactions from all involved parties to reach a conclusion as to the future of the project.

As this is such a strict, somewhat secretive and regimented industry we found there weren't many stakeholders, however, the ones we did isolate were more than capable of delivering the information we needed, sometimes answering questions or giving suggestions we hadn't thought of. WADA were the obvious first choice to gather information, the only way to contact them was through their website to await a response. There was no possibility to interview anyone within WADA as 2016 was a busy year for them with the Rio Olympics and other sports events to monitor, but we were given the option of a survey, which we emailed through to be directed to the most appropriate person.

ASADA and Drugfree Sport NZ both have an education-related email in the "contact us" section of their website, to which we sent an email briefly explaining our project to enquire as to whether we could organise and interview with an appropriate member of staff. We requested to interview a member of staff that was specifically involved in the sample collection aspect of the companies. We awaited a response from their education 
administrators who then promptly put us through to the appropriate people. ASADA politely directed us back to Drugfree sport NZ, saying that they could answer all of our questions. This interview was conducted over the phone and recorded for future analysis and reflection.

On the WADA website is a list of all the accredited labs worldwide. Full information is given about these labs - there location, phone number, continent they're in, the region of the world they manage, and the most useful part was a direct email to contact the directors. We sent an email to the Director of ASDTL, which is the only accredited lab in all of Oceania, with an information sheet and a request for an interview with a member of staff that could answer our questions which we had sent in advance. This was the most efficient means to get into contact with this stakeholder as the lab is based in Sydney and by communicating via email we were able to organise and book a time for the interview with a relevant contact. Initially, we were going to travel to Sydney to conduct the interview in person, however the contact was unavailable during the time we could travel so the interview was conducted over the phone and recorded for future analysis and reflection.

Our next candidate for interview was an ex-global level competitive athlete, now turned an esteemed professor of sport, coach and sports event organiser. We were put into contact with this source after a presentation of our project, where we openly asked the audience if they had any contacts to aid in the development of the project. We swapped emails with an audience member who then asked this professor to contact us. We organised and booked an interview, which happened over the phone and was recorded for future analysis and reflection.

We generated a generic survey for coaches and athletes to fill in and send back. Initially we wanted local professional teams, such as the Wellington Phoenix and The Hurricanes, however they did not respond to any of our emails so we assumed they either weren't interested or were too busy. This meant that we had to call on other athletes, reaching out to them through social media and direct e-mail. Fortunately, the ones we did contact we able to give invaluable insight in their responses to our questions.

We contacted TDTC through their website to explore and understand their development strategy and business model. We were put into contact with the managers of both the 
Auckland and Wellington branches of the company where e-mail was our primary method of communication. However, during the exploration of this project Auramer Bio received a grant to complete work more engaged in this illegal drug testing field, so we decided this was outside of the Rapid Athlete Testing avenue. The lead researcher passed on the contact information that had been acquired and left this source so not to jeopardise the other project.

The information provided by each research participant gave valuable insight into the potential applications for the device within the market of testing for signs of drug abuse and doping in sport. Each interview was recorded and transcribed to truly visualise the responses and provided the opportunity to look for, note down and re-evaluate our assumptions. Through the research participants' feedback, we learned more about the war on doping, the athlete's perception of prominence of drug abuse and the history of aptamer-based biosensors within the industry. We examined this feedback from our research participants with our lead technical scientist on the project, as well as the CEO of Auramer Bio to discuss their thoughts of the research so far and relay any recommendations or concerns. This was an important meeting to determine the progress of the project and the future direction it could take and to culminate ideas based on these findings.

After this critical meeting, the research methodology remained the same but the goal of the research took a slight pivot, as it became apparent of the limitations for our device entering this rigorously structured market. Our initial research methodology sought to uncover whether our potential stakeholder groups were interested and whether they could perceive enough value to integrate our device into their framework. The device has the potential to be incredibly disruptive within this industry and promotes a lot of value, although after reflecting on our approach and ruminating over recommendations made by our lead technical scientist, CEO and from audience members present during our final presentation, we realised we may have been over-ambitious in our attempts to enter the market by our current means. The main limitations became apparent because of the information gathered within our interviews and research, and so we changed our objectives to determine whether a suitable starting point could be established. Recommendations made by our lead technical scientist suggested that we should investigate another way to utilise the device within the drug abuse and doping detection industry. From this suggestion, we reconnected with a few 
of our previous research participants to discover their thoughts on our new direction by conducting another interview. These interviews yielded some interesting results and proposed several new ideas that could bring the device to market within this industry. We did this so that we may still find a way to enter this market with our device, or determine that instigating change with our device on such a large scope would prove inviable.

\section{Challenges and Resolutions:}

There were numerous challenges and limitations of the research that had to be overcome or navigated correctly to successfully connect with the right people and obtain the vital information we needed to assess the commercial viability of our device. Primarily, as this project stemmed from a University Master's course, the University's Ethics Committee had to permit and endorse the research before interviews and exploration could formally begin. It was within the ethics application process where the challenges and limitations withheld the momentum of the research. Firstly, there was the limitation where the ethics committee only held a meeting once a month, and if an application isn't in two weeks before that meeting - even though it's in a digital format - the application will not be reviewed and is then forced to wait until the next meeting. Secondly, within this process our application for ethics approval was removed from the line up by the admin to update approximately 5 answers, all of which seemed trivial and could have been picked up had it been reviewed by the committee. At this point, we were under the impression that it had been reviewed and that these were the changes required for approval of the research. We later found that this wasn't the case and that after making the changes we were not eligible to have our application reviewed in that month's meeting. This pushed the starting point of the research back a further 6 weeks as we had to wait for the next meeting and make a few alterations to the answers within our application. From beginning to end, the ethics committee process took 3 months to grant approval, approximately a quarter of the time allotted to complete the Masters Course. This created a huge barrier as the Masters project had already changed from exploring a previous project, which left 6 months to formulate and gather the research as well as write the final report. Other members of the Masters course also had issues with ethics approval and more than half received an extension of up to 3 months.

Another challenge and limitation of the research lay in the fact that it comes from an exploratory course and that the contacts to reach out to conduct research were not 
established like other Masters courses at the University. Due to this, a large crutch lay in the fact that we effectively relied heavily on all of our research participants being interested enough to participate in the research. Within such a regimented industry, if we had not gauged enough interest to create and undertake an interview with the appropriate parties, we would have been stuck at a dead end and it would likely have culled the project in its infancy. There were many limitations in what participants could also disclose within our research as it does hold commercially sensitive information. This was overcome by having research participants sign non-disclosure agreements and state whether they wished to be named in the final report. Also we found that because the research came from a Master's course, all of our research participants were much more friendly and willing to be interviewed.

Furthermore, during the interviewing portion of the research it was a challenge to properly convey the arrival of the device to research participants as it was in its lab component stage and we did not have any indication when a prototype would be introduced. This became a challenge as the research participants gained interest in the possibility for the potential of this device, yet we could not give a clear indication of when we could intend to enter the market. We realised this limitation though, and anticipate using these established contacts in the future for the true development of the project.

Another challenge of the research lay in keeping in contact with Auramer Bio to maintain the relationship and be able to relay the findings of the research as new and prominent data presented itself. This was managed somewhat effectively by the lead researcher organising meetings when possible, but due to the time constraint on the research caused by the delay in ethics approval, a lot of time had to be dedicated more to research and findings.

Finally, the biggest challenge and limitation came from time constraints on the research. These were imposed by the University, the result of the ethics committee, the research participant's schedules as well as commitments that the lead researcher had to adhere to. Due to the combination of all of these time constraints, the research had to be conducted quickly in order to be completed successfully. Overall, this had no impact on the quality of the research, it simply meant that the time management of the research had to be impeccable, which was a challenge in itself. This challenge was managed by referring to our 
Gantt chart and simply trying to compact more research in the start of the time period so that reflection and writing of the final report could be completed later. 


\section{Findings and Analysis:}

\section{Exploration of Current Behaviours of our Stakeholders to Evaluate our}

\section{Assumptions:}

Throughout the exploratory research phase process of this project, we sought to either confirm or invalidate our assumptions in order to have a clear vision on where we could potentially stand within this market.

Firstly, we had to understand the current behaviours of Drugfree Sport NZ, WADA and ASDTL. We also had to gain an understanding of coaches and athletes experiences with the current sample collecting and testing methodologies. By doing this, we could then gauge if any shortfalls were apparent in their current behaviours that we could take advantage of with our device.

\section{Drugfree Sport NZ:}

Within our interview with our research participant from Drugfree Sport NZ, we learned about the current process surrounding sample collection, what happens to the samples after collection, current costs involved, the Athlete Biological Passport (ABP) programme and conditions maintained around the samples for quality control. The current techniques for sample collection as outlined on the Drugfree Sport NZ website elude to an " $A$ " and " $B$ " sample bottle to be filled with urine, or a blood sample be taken and packaged into a tamper-proof kit and then sent off to ASDTL. However, the process isn't that straightforward as we learned that the urine samples are frozen and sent to the Drugfree Sport NZ office where they are sent to ASDTL on a weekly basis. This can cause issues when it comes to the detection of certain substances as the freezing and thawing can affect the detection thresholds of a substance within a sample. Blood samples only have 48 hours to have the tests carried out on them before they start to denature and become a redundant sample. Due to NZ being remote and blood samples needing to be maintained within a certain temperature range, blood samples wouldn't make it to Sydney on time for the blood tests to 
be carried out. Based in Auckland is a forensics lab that has WADA accreditation to carry out the blood tests for the athletes.

We also learned of the ABP programme and the costs involved when it comes to sample collecting and testing. Sample collecting and testing is a constant operation for Drugfree Sport NZ, where the off-season or out of competition testing pool lies at approximately 150 athletes. In the on-season or in competition, any athlete can be selected for sampling and testing. The ABP programme aims to create a profile for an athlete to therefore monitor for any changes as well as aid in research. There has been a lot progressive research into dry blood spot analysis for this industry, blood reveals certain markers of substances such as EPO, whereas urine highlights a steroidal profile.

\section{WADA:}

Next, we had to understand WADA's current behaviours within the industry from both a practical and legislative standpoint as we needed to apprehend how they guide the industry. WADA has 34 accredited labs across the world to conduct the tests for signs of drug abuse and doping in sport, which to us seemed like a small amount. However, after our survey came back from WADA, we found that this is actually considered a large quantity of labs, given the technicality of the analysis and the limited number of samples. It is best to have a good spread of labs across the globe so that all labs can receive a sufficient number of samples to maintain expertise in the techniques used and to make the overall cost of testing much more efficient.

Figure 4::Location of WADA Labs, 2017

WADA's prohibited list is the international standard for identifying forbidden substances in sport. The prohibited list

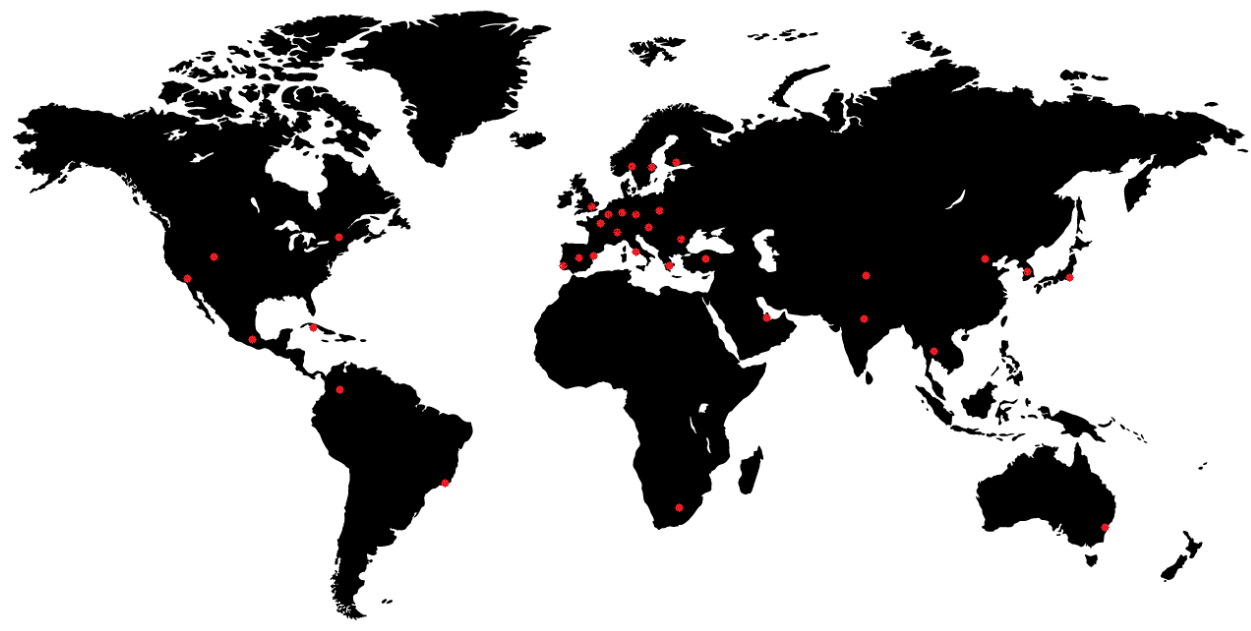


serves as a key component of the international code which promotes the global harmonization of the anti-doping in sport movement. WADA's prohibited list is broken down by category and is updated annually after a consultation process by WADA.

Substances that are considered for the prohibited list must either have the potential to enhance performance, endanger the athlete's life or breach the overall spirit of sport and sports conduct.

\section{ASDTL:}

Finally, to understand the current behaviours within the official testing chain of the industry, we needed to understand ASDTL as a business and their internal procedures. Through our interview, we learned about the many types of testing they conduct and how they interact with their clients and athletes. We also learned about the types of substances being abused and their role in the detection of these constituents, as well as their history and operations.

ASDTL was formed after the previous International Olympic Committee (IOC) lost their accreditation and Australia was looking to organise their next Olympic bid, which requires a thorough pledge of how they will complete drug testing and their stance on drug abuse and doping in sport. In 1999, WADA was formed and all of the IOC labs became WADA accredited labs. For a new to lab to become WADA accredited, it has developed to be a difficult process. The lab needs to be fully set up with all of the equipment required, including the expensive machines to conduct the more intricate tests such as EPO or Human Growth Hormone (HGH). A new lab must also receive a letter from a national WADA agency such as Drugfree Sport NZ, saying that they will send them at least 3000 samples per year as well as receive enough financial support to operate the tests and equipment. Once all of the setting up has been completed and the requirements have been met by a new lab, they may enter the WADA accreditation process, which can take approximately 18 months as the proficiency tests require are carried out only a few times per year. A team of around 7 people from WADA visit to examine security measures, finances and proposed research programs as at least $7 \%$ of the labs annual budget must be spent on innovative research and not mere development. Once accredited by WADA, the lab is then restricted to only testing athlete's samples. ASDTL does some testing for the defence forces, but requires WADA approval to do so. 
As most of the samples for testing tend to be urine, where ASDTL and other WADA labs look for excretion metabolites of substances, they rely hugely on excretion studies to draw conclusions. If the study has been funded by the pharmaceutical company that creates the drug, then further peer reviews must be explored to ensure accuracy and fairness when testing in sport. This is especially true for new steroids as they must first be made and tested for metabolites in order for detection methods to be formulated. Steroids are the most prominently abused substance by athletes in the sports industry.

In terms of volume, ASDTL tests approximately 5000 samples per year, which is not considered a lot. This is due to the geographic layout of Oceania where there isn't a lot of country mass or a huge population. The fact that the samples must also be couriered to ASDTL as it is the only accredited lab in the region further adds to the costs involved, which is why ASDTL is a government funded lab. In order to be solely financially viable, an accredited lab needs to test approximately 20,000 samples a year, including having a scale of economics for the number of samples being tested - one or two samples will cost more than sending in a bunch of samples. As some of the machines can cost around \$450,000; a lab will want to run enough tests each year to make it affordable for the clients.

When it comes to the cost of tests for the labs, there are two main programs that the testing can be categorised into - in and out-of competition testing. Out-of competition testing is for substances that are banned all the time, for example anabolic steroids and diuretics. In-competition testing also seeks to detect stimulants that can be used to have a direct effect on the competition. Every sample is tested for in and out-of competition substances depending on what is required for the clients. The initial screening alone is run on a 96 well plate which has 4 control wells, meaning 92 wells of sample can be tested in a single run, this process usually takes about a day to complete. On top of this is more specialised testing for substances such as EPO, which is a large molecule but is hard to test for as the body produces it naturally. EPO is present even in small samples, so a typical test for EPO consists of taking $10-15 \mathrm{~mL}$ of sample and concentrating it down to $50 \mu \mathrm{L}$ which is then run on a gel to visualise the sample, the testing for EPO averages around AUD\$260 per sample on top of normal testing costs. The more intensive testing procedures generally involve substances that the body produces naturally, such as testosterone, and therefore the detection aims to determine the difference between the natural levels and those 
elicited by the pharmaceutical product. These more thorough methods of testing naturally cost more and increases the workload of the labs and therefore the overall cost of sample testing for the clients.

In the lab, the procedure for testing and storing samples changes depending on the matrices obtained by the sample collectors. For urine, which is the predominant sample matrix obtained, both the A and B sample bottles are checked that they are untampered with and that the paperwork matches up with the sample. The B bottle is stored in a freezer, whilst the A sample bottle is opened up, has an aliquot taken from the sample for screening and analysis, the A sample bottle is then placed in the freezer. The screening will commence based on the clients' needs and whether the sample tests are for in or out-of competition and if more extensive tests such as EPO testing is required. When it comes to the results, if a negative result is presented, then that result will be reported to the client. If a positive result is presented, then another aliquot is taken from the A sample bottle and a detection test specifically for the prominent substance will be conducted for confirmation. If another positive result occurs, a report will be sent to the client who then contacts the athlete in question, who has the right to inspect the $B$ bottle sample testing. 


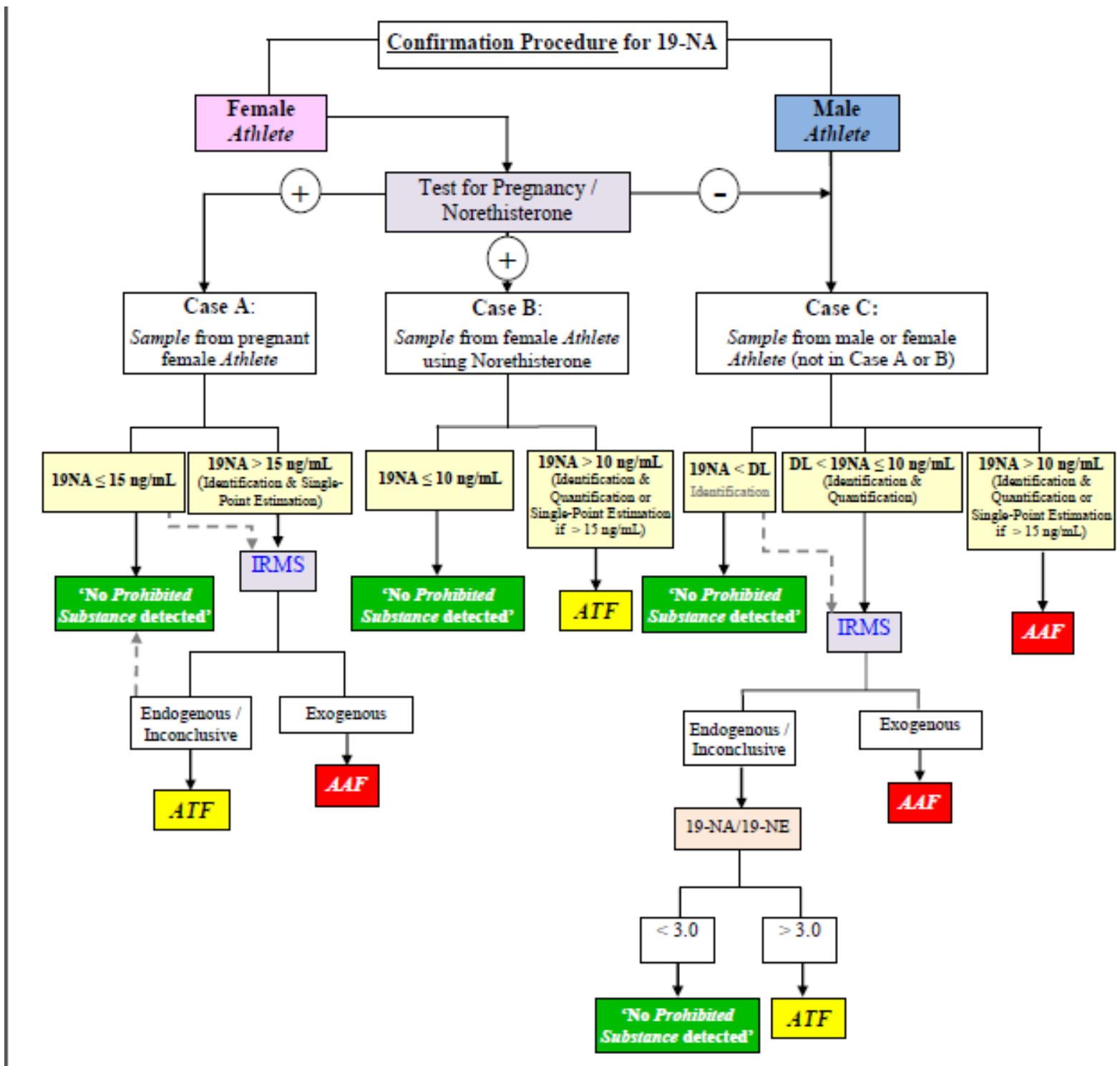

Figure 5:(Technical Flow Chart Confirmation Procedure, Courtesy of WADA Technical Document td2016na-en.)

\section{Coaches and Athletes Perceptions:}

Four our project to truly understand and adapt to fit a niche within this rigid industry, we must also understand the experience and perceptions of the end users. For this we asked for experiences on the current methods and their perceptions on how the industry is currently run. From this we found that our coaches, who had also been personally tested, had trained athletes that had been tested and strongly believe it is the right thing to do. The current drug testing process is perceived to be a breach of privacy for the athletes but is also considered a necessary evil to maintain fairness within sport, but it is wholly considered in their experience to have been conducted in a very professional manner. 
What is perceived to work well about the current process of in-competition testing is the selection process, specifically that an athlete can turn up at the finish line and not know whether or not they are going to be tested and caught. However, there is always the general concern over the robustness of the testing as stories of athletes bypassing the tests or buying out the sample collectors has plagued the drug testing in sport industry for many years. Although sample collecting and testing in New Zealand and Australia is perceived to be largely more professional and robust in terms of ethics and results.

\section{Stakeholder Barriers to Changing Behaviours:}

After a thorough investigation of our stakeholders current behaviours, we then needed to investigate if there were any barriers to changing these behaviours. This exploration was most important for Drugfree Sport NZ, WADA and ASDTL as they would be the groups that we would need to convince in order to initiate a change of behaviour that could lead to the adoption of our device within this industry.

\section{Drugfree Sport NZ Barriers:}

Drugfree Sport NZ issue the sample collection process, so we needed to determine whether they had any barriers, such as the purchase of new equipment or any legislative restrictions, that may prevent them from adopting the use of our device within this industry.

The New Zealand law for taking samples from athletes for drug abuse and doping purposes refers to adopting the WADA code. From this, we can infer that if the WADA rules could change or adapt, then so must Drugfree Sport NZ's policies and procedures. The current rules are more focused around the sample collection process, transporting of the samples, the lab tests themselves and the management of the results.

A barrier to using our device as an onsite testing function revolves around the fact that in an open environment, microbial infection or contamination of the sample can affect the results of analysis. Accuracy is essential within this industry and for a start-up company with a new device, we cannot deliver false-positives. If our device can be used as an indicator tool of which samples to send away for more formal testing, there could be a cost saving produced and more efficiency added into this system. 


\section{WADA Barriers:}

The largest barrier WADA faces when it comes to introducing new methods into this industry is the validation that the proposed technology can provide the results required to meet the accreditation standard. WADA has previously invested in the same technology that our device runs on, with previous projects either falling flat or the companies themselves falling into financial ruin. It is due to the previous failed attempts to use aptamer-based biosensors within this industry that WADA may be reluctant to give the device a chance without sufficient evidence of its effectiveness and efficacy.

\section{ASDTL Barriers:}

The barriers for ASDTL in potentially adopting our device lie in the already established scientific methods and the processes in place. For example, a well-known and established set of antibodies are in place for testing for EPO as they are well studied in their detection of the specific metabolites. In order to make a change to the system - even if it is simply changing the antibody being used within the tests, evidence needs to be presented to WADA to show the clear benefit of making said change.

ASDTL has also had previous experience with aptamer biosensors as a potential medium for testing. A company stemming out of the UK aimed to use aptamers to replace antibodies for testing, but their funding lapsed on the first phase and so the project didn't go anywhere. ASDTL is still quite interested in aptamer technology to replace antibodies as they could improve the sensitivity of the tests and are more effective in the long term as the antibodies used could run out. Although there is nothing preventing the use of new methods, ASDTL are limited by what WADA will permit so cannot simply integrate without sufficient evidence of the validity of the device and WADA's permission.

\section{Stakeholder Perception of Our Proposed Product/Solution:}

The last stage of analysing and interpreting the commercial viability of our device with our potential stakeholders involved gauging their perceptions on the possibility of its value to the industry. It was here that we could assess the true value of our proposed device and whether the direction we envisioned for the device could be viable. 


\section{Drugfree Sport NZ Perceptions:}

Drugfree Sport NZ organise the sample collecting, transport of the samples and the eventual prosecution of those that breach the rules within New Zealand for this industry. As we could perceive value in the use of the device as an indicator tool when sample collecting, it was imperative that determine whether they shared the same opinion and if they had any further insight.

Our research participant at Drugfree Sport NZ could perceive a lot of benefit for having our device onsite as the sample collectors could then quickly screen a greater number of athletes and then use the information generated to select which samples needed to go away for more rigorous testing. They could also perceive the value that the device could operate over multiple matrices (blood, urine etc.) as often when an athlete has been selected to produce a sample, the sample collector must stay with them until they pass the urine sample to ensure no tampering of evidence has occurred. This also involves checking the genitalia and watching the sample be passed into the sample bottles, which has been described as "rather invasive" and "awkward".

Overall Drugfree Sport NZ's perception of our device revolved around whether there is a big enough cost saving to sway the necessary people. If the device could collect and screen multiple samples at a lower cost there is a clear benefit, but also if only certain samples were sent away for more formal testing then there is further advantage in terms of cost savings. Lastly, as long as the device can detect substances in blood as effectively as urine, then it provides a clear value as urine collection can be very time consuming, most athletes would prefer not passing urine in front of someone so blood is possibly the best candidate for screening matrices in this situation.

\section{Coaches and Athlete's Perceptions:}

The coaches and athletes are the members of this industry who are at the receiving end of the sample collecting and drug testing and whom are often overlooked in terms of how the process affects them and their participation in sport.

In asking about our device, there was a suggestion that the athletes and coaches could have a reference number to effectively "track and trace" the progress of their drug tests. This is because they generally don't hear back about the results unless they have tested positive 
for something, which can sometimes add stress and hinder performance. This is an idea we can incorporate for any more formal tests that the device has determined needs to go to an accredited lab.

In exploring the possibility that coaches and athletes could use the device as a way to "selfmonitor" themselves as they train, we found a perceived benefit as supplements are not regulated by the FDA and so can contain more substances in them than what is on the label and some of these substances are on the WADA banned list. In order for the device to be truly useful, it must be portable, accurate and reliable in the delivery of its results. However, self-monitoring shouldn't be totally relied upon as every device requires care, maintenance and calibration. The coaches and athletes recognised that as far as the benefit for testing where you are not required to drop your pants and be observed passing a sample, formal testing will always provide the most trusted and robust results.

Lastly, a clear benefit could be perceived if a coach could own a device to check his athletes, as formal tests cost approximately $\$ 700 N Z D$ and then a further $\$ 500$ for subsequent tests. If the device was accurate, portable, reliable and could be used in even the most remote areas whilst reducing the costs involved with drug testing, then this stakeholder group holds the opinion that the device could be a winner.

\section{ASDTL Perceptions:}

ASDTL are the only WADA accredited laboratory in Oceania, so their insight into the possible value of the device was worth its weight in gold. With the feedback presented by ASDTL, we have been able to hone in on where exactly the device could be used within the accredited labs to increase efficiency and cost-savings for the lab and industry.

When we asked for their perception of our proposed idea for the device and its use within ASDTL, we learned that whilst it is a possibility to use the device how we intend, it would only be a short-term solution. This is because ASDTL, WADA and other accredited labs are changing their perception of substances and how they undergo the detection of these substances. This shift has occurred because of the changes and evolution of mass spectrometry and a better understanding of what can be tested for. Furthermore, WADA and the labs involved operate on urine, which means the results they base their decisions on rely on excretion studies. When it comes to Olympic-level athletes, they are genetic outliers 
and it is hard to categorise them effectively with those within the normal population. This is one of the factors that has drove the shift from simply detecting substances to looking for noticeable differences in a specific athlete's haematology.

ASDTL also expressed their biggest concern for using the device in the manner that we had originally intended would involve how well it could compete with the current methods in place. For the current In and out-of competition testing, the samples are screened using liquid and gas chromatography to examine the samples for evidence of anywhere between 200 and 650 different substances. As an aptamer-based biosensor, it would not only need a suitable library or array of aptamers to detect for all of the possible substances, but it would also need to allow for the difference between endogenous and exogenous versions of hormones as the structures are very similar but the substances themselves are abundant at vastly different concentrations.

As a more practical alternative, ASDTL could perceive value in the use of the device for the more expensive tests such as EPO or peptide hormone detection. EPO detection requires antibodies, which in the long run could run out, whereas aptamers can be generated relatively quickly. The more expensive tests not only cost more to the client and to conduct for the lab, but they also take a lot more time. This means that if the device could prove to serve as an accurate and reliable confirmation analysis, then it could be hugely advantageous to the industry as a whole.

Another insight provided by ASDTL's perception of the device lay in the fact of how important it is to work with a WADA accredited lab when trying to enter the market. This is because outside of the industry and community of testing for drug abuse and doping in sport, there are a plethora of assumptions that are completely wrong. By working with the lab to work on a proposed idea, a company can have access to information about the industry, the research being conducted and how they can therefore integrate now and for the future.

Lastly, ASDTL noted that whilst our device works well across matrices, we needed to consider the geographical limitations of Oceania and the fact that Urine is a much more sensitive matrix for long-term metabolites and is a better medium for testing within the lab. We must also keep in mind that the future is not in looking directly for the metabolites, but 
looking for changes within the ABP which will give off biomarkers. If our device could be used as an indicator to detect the fluctuations in these biomarkers, then pursuing only the necessary athletes for a urine sample and more formal testing could be a profitable and viable future.

\section{Authentication of our Assumptions}

The overall goal for the Rapid Athlete Testing Project was to assess the commercial viability of Auramer Bio's aptamer-based biosensor within the drug abuse and doping in sport industry. Within the course of the inception of the Rapid Athlete Testing project we made many assumptions in terms of the market development strategy and market opportunity. Our assumptions lay in the aptitude of our technology and the potential disruptive capability it may have on our proposed market and industry. Our main assumption was that our device could provide enough value to initiate a change in behaviour within this largely scoped but highly organised industry and be a profitable endeavour. We assumed that our quick turnaround times, accuracy, proposed portability, multiple matrices for sample collection and cost-efficiency would make for a quick integration into the industry, as well as the assumption that we were exploiting a niche within this market that our customer segment may not have realised they had. We assumed several potential paths to market for the device to be used in either the lab or onsite by the sample collectors. It was within our research that we were able to test our assumptions and theories and gather the information required to make a more informed decision as to the future direction of the project.

Our assumption that this market yearned for portable, multiple matrices testing and quicker turnaround times was confirmed by all of our research participants. Our pool of athletes and coaches explained that by having a long test period, the pressures on the results looming over the athletes can have a detrimental effect on performance. This pressure at the internationally competitive level where athlete's results times can vary by milliseconds, can have an impact on competitive edge which may be the difference between winning and losing. Also for athletes, when selected for screening, they have to have a sample collector by their side at all times, awaiting the athlete to pass urine into the two sample bottles so that it can be packaged into a tamper-proof kit and sent away to the lab. If a portable sample testing device were present, the result turnaround time would be drastically 
reduced and, depending on the result, could alleviate the stresses and pressure associated with the sample testing process.

The sample collectors also spoke highly of the need for portable, multiple matrices testing and quick-turnaround times. For example, if a sample collector must test an entire rugby team, that's 15 primary players and 7 reserves that they have to wait for them to pass urine, package into kits and send away to the lab and this could take several hours. It was found from both the athletes and the sample collectors that blood is much more time-efficient, perceived to be less invasive than being watched passing urine and means that a sample can be collected within 5-10 minutes as opposed to a potential several hours per athlete when waiting on urine. Our research participant from ASDTL also expressed the grave benefits our device could employ in the terms of being used to replace certain lab equipment. Currently, the average cost of testing each athletes sample is approximately $\$ 700$ ! This can go higher if more stringent testing is required. By implementing the device in the labs, this could lead to an overall cost saving by reducing the amount of materials and technical equipment required to run the tests as well as a saving on the labour required to manage and maintain observation of the tests. Overall, by conducting research with our participants we were able to confirm that whilst it is a stretch to conclude that our device is the answer to their problems in terms of portability, multiple matrices testing and quick-turnaround times, all parties involved could perceive a lot of value in these qualities. We therefore can interpret that as all of the main participants could perceive a lot of value in these qualities, that our device can accommodate a need in the market that we originally believed were assumptions.

We made the assumption that a highly accurate, fast device that bolstered cost efficiency would naturally be taken with open arms and integrated quickly into this industry with high praise. However, upon testing this assumption we discovered more than anticipated. Firstly, that a "mobile lab" is already in use and has WADA accreditation for use in the ABP program, this is a good sign as it confirmed our assumption that a device itself can gain accreditation. WADA documentation requirements refer to "validation of the method", which confirmed another of our assumptions that in order to gain accreditation we would need to conduct sufficient testing and have our work peer reviewed to prove the capability of the device. This would be a logical and necessary step for the project anyway as it could 
be used as evidence of accuracy and credibility for other avenues Auramer Bio is exploring for the device.

Secondly, we learned that when it comes to larger events such as the Olympics, the current testing methods mean that the 34 WADA accredited labs worldwide are run 24 hours a day and more WADA lab staff are distributed to help with the load. This means the whilst the testing methods are obviously more inflexible, the increased load has a large impact on the testing facilities which could be mitigated.

Lastly, it became apparent just how quickly evolving the drug testing in sport industry has become and what a vicious cycle it is. WADA has to adapt their behaviours to account for the athletes, who then in turn adapt their behaviour to attempt to surpass WADA's progression or improved testing. For example, urine used to be the only sample collected to test for excretions of the abused substances. Now both blood and urine are tested for traces of the substances themselves but also for the effects of the substances. Occasionally a new testing technique is implemented for the first time at major events to test for ne and upcoming methods of cheating. (eg EPO testing at the Sydney Olympics.). New drugs are also brought to WADA's attention all the time, be it directly from a pharmaceutical company, as an anomaly found whilst testing a sample that is then investigated further, or even athletes admitting to using them. A lab with expertise will usually then develop a test or technique for detecting this specific substance. This confirmed that there is a need within the market for a cost-efficient, accurate and time-effective device as they need to produce accurate results, occasionally with a high-volume workload within a reasonable timeframe so not to blow their individual budgets on labour charge, materials etc. The interesting point presented here is that a new substance may be presented an expert lab must then create a technique to test for this. Our device uses aptamers, so we would only need to produce a complementary strand of the substance, so a detection method can be produced quite quickly, adding further cost-effectiveness to the testing portion of this industry.

We initially made the bold assumption that, after gaining accreditation, our device could have the disruptive power to circumvent the lab step entirely and produce all of the required results onsite. This assumption proved ludicrous to our presentation audience, WADA, Drugfree Sport NZ and ASDTL. Whilst our device realistically could circumvent the lab stage and produce the results onsite, this would cause a lot of disruption for WADA, who 
has spent nearly the last 50 years developing policies and procedures to combat drug abuse and doping in sport. This would also mean that the WADA accredited labs such as ASDTL wouldn't have anything to do as most of them are so specialised for the ABP program and testing athletes samples, that they would have to be shut down, causing a large loss of jobs and experience within the industry. When it comes to producing results onsite in the hands of the sample collectors, they don't have the authority to arrest any athletes or search their belongings for evidence, so this idea falls through logistically, as police can't be present for every single sample collection and result, especially at big events where other matters would take priority. All of these ideas caused the pivot in direction for our device and how we planned to enter the market. Our assumption was quite literally destroyed, but it did open the idea for a starting point to use the device as an indicative tool to separate the "potential abuse" samples from the "definitive evidence" of abuse samples. The idea to use it as an indicative tool to separate the samples and promote a cost-saving by only sending the necessary samples to the lab proved to be a much more popular idea by all of the research candidates.

Further assumptions that were unaccounted for were presented whilst interviewing one of our coaches. We assumed that the teams and coaches perceived the sample collecting and testing as a cumbersome and unwanted endeavour as they are time-consuming and add an undesirable pressure to the sport. This assumption proved only partially true, as we only viewed this from a business and efficiency perspective. This particular coach that we interviewed explained that whilst the sample collecting and testing added a sour element to sport, they are a necessary step in order to provide a level playing field for all athletes and ensure that an individual can revel in the glory of their win.

When it comes to the organising of an event where large sums of money or prizes are available for the winners, there is therefore a larger incentive to gain a competitive edge. For event organisers who want to run a fair and successful competition, this means they need to drug test. This then requires contacting their national department that is in charge of conducting the collection of samples (For New Zealand, this is Drugfree Sport NZ) to organise sample collectors for the event. As there is already a large cost of $\$ 700$ per sample to be tested, alongside the cost of hiring the sample collectors as well as sending the samples to the labs, running an event will currently incur a large cost upfront. Our idea that 
our device could presumably now be used as a cost-saving indicator tool for samples collectors at such an event was received well and further aided the decision for the pivotal change in direction for the device and the project. We then made the assumption that this could be a viable direction as a starting point for the device within this industry. We later relayed this idea to our research participants at Drugfree Sport NZ and ASDTL who confirmed that this would make for a good starting point and could be a suitable way for Auramer Bio and the device to be a proven asset within the industry.

\section{Project Opportunities:}

An unexpected opportunity arose for the project upon discovery that our research participant from ASDTL had completed previous academic work on the same technology that our device operates on. This was the primary drive for this participant's involvement in the research and led to the discovery that WADA had invested in this technology before. In the past, WADA had given grants and funding to companies to develop their aptamer-based biosensors for use of testing athletes for signs of drug abuse and doping in sport. However, these projects had previously lapsed due to limitations in technology at the time, the companies going bust due to economic recession, or the projects being axed by the companies involved altogether. Due to these past examples of aptamer-based biosensors lapsing for WADA, naturally there is a general distrust that this technology could be used, let alone gain accreditation within this industry.

Our research participant presented an idea that once Auramer Bio had developed a functional prototype, that a series of tasks and activities could be completed to confidently prove the capability of the device. These would be based and limited to the Oceanic region as it must be carried out within their jurisdiction, but would ultimately serve to bolster the credibility of the device. Once these tasks had been completed and showed without a doubt that the device wouldn't produce false positives within samples, ASDTL could then present the device to WADA and use the evidence developed from the tests and tasks to hopefully gain funding for further investigation and peer review. After the peer reviews and revision of documentation, the device could then be put forward for accreditation and eventual integration into this highly-regulated market. 
The unexpected opportunity given from our research participant has highlighted the possibility for a potential path to market. Auramer Bio would need to prove the accuracy and efficacy of the device in order to even consider approaching WADA anyway, but with the guidance and use of our new contact, we will stand a better chance of success. Although the result is not guaranteed and a lot of work would be required to obtain the necessary WADA accreditation, our device and project at least have a "foot in the door" contact to which we can utilise for the future development of the device's credibility and hopefully the overall success of the Rapid Athlete Testing project. 


\section{Assessment of the Viability of the Project:}

The main objective of the Rapid Athlete Testing project was to assess the commercial viability of Auramer Bio's aptamer-based biosensor within the industry of detecting signs of drug abuse and doping in sport. We decided New Zealand and Australia would be a suitable starting point as there is only 1 WADA accredited lab in the area and in a business sense there is more signal and less static from competitors and other markets. Firstly, we needed to analyse previous opportunities with similar technologies, investigate the market to determine if there is a sufficient need for our device, either validate or invalidate our assumptions and finally gain some form of confirmation from stakeholders within the industry. After achieving these goals, we could assess where we stood in terms of entering this market with our device and whether it would be a profitable and successful venture.

Our initial idea was over-ambitious and it was because of our assumptions that we thought our device could be put in the sample collectors' hands and totally circumvent the lab stage of the process. However, in theory this is grand but the device was still in its lab component stage and a prototype had not been developed. We decided that a commercially viable prototype was definitely not ready or able to replace the 34 well-outfitted and equipped labs around the world. From this we decided that a more suitable starting point needed to be explored in order to bolster the credibility of the device and gain WADA accreditation. We needed to isolate a point in the industry where our device could be used, whilst obtaining evidence of the devices' capability to present to WADA.

Our next idea was that we could start smaller and still use the device with the sample collectors, but to be utilised as more of an early screen to decide which athletes required more formal testing, rather than sending every single sample away to an accredited lab. Drugfree Sport NZ and Auramer Bio could perceive the cost-saving benefit of having a selective device that could be used to differentiate between samples, creating a saving on both testing costs and the transport of the samples to the accredited labs. Our Coaches and Athletes were also on board with the idea as long as it could prove to be accurate and reliable, this is because no one - athlete, coach or company that has promised an accurate 
device - then be accountable for a false positive result on a banned substance. This was the main idea and goal of the project until we interviewed our research participant at ASDTL who shed light on the vast extent of substances that they test for just in their initial screening phase.

After our interview and discussion with ASDTL, it was apparent and painfully obvious that our previous goals and assumptions had been far too ambitious and that we were after all, competing not only against other companies but with the processes and legislation already in place, with a device that was not ready to be released to market. It was within this interview that we found a suitable starting point for the device within the industry as our research participant had highlighted previous work using aptamer technology for testing to detect the more expensive and complex molecules such as EPO. The more expensive tests which use the current methods require a $\$ 450,000$ machine and a lot of preparatory work, lab technician time and the cost factor of care and maintenance of the machine itself. The opportunity for the device to be used in place of this arduous and expensive machine which also requires antibodies, of which may run out at some point where aptamers will not, has the potential for a drastic cost saving for the industry. This is because it would be incredibly hard to compete with the mass spectrometry screening being utilised currently to initially assess the samples, as well as the technical limitations of aptamers being able to screen for a multitude of different substances at the same time. It was also within this interview that we were offered the possibility of completing some work with the device once it had been sufficiently developed, confirming both a need and the commercial viability of the device.

\section{Opportunities and Barriers of the Project:}

The opportunities and barriers of the Rapid Athlete Testing project surrounded the overall structure of the industry, the legislation and testing policies in place and the persistent pressure athletes in the higher levels will always face that causes them to turn to substance abuse in sport. There were also opportunities and barriers present within the financial and legal aspects of the project.

Primarily, the main barrier to entering this industry resided in the fact that WADA as a governing body has to permit every act within the industry in order to maintain fairness and security throughout all of its constituent labs and national government sporting agencies. 
What this meant for our device is that we must develop it to a functional prototypical standard, perform multiple tests with it to prove its efficacy and the effectiveness of its use for the industry. Following this, the device would require multiple testing and peer review to provide sufficient evidence on the performance and capability of the device. However, these stages would be required regardless of which industry the device was intended to enter due to it being a new and innovative device that would be proficient in many areas. Although, the fortuitous opportunity to work with a WADA accredited lab to utilise the device and bolster its credibility with tests related directly in line with the operations of the industry was an incredible prospect, which also truly mitigated the burden of endeavouring to convince this industry that our device could work just as effectively within its framework. Our barriers to entering this industry have been drastically diminished in recognition of our research participant and ASDTL's gracious offer, as we now have a "foot-in-the-door".

Our next barrier was formed from the limited timeframe set in place to explore the viability of the project as it was in conjunction with a Masters course. Due to the exploratory nature of the course, it was expected that a researcher be able to work as a pioneer to map out an industry, find a market need and provide a potential product or solution to that need. Conversely, because the of the time limit, true exploration was nigh on impossible without a strict time management scheme. Furthermore, the time lost awaiting ethics application not only threw out the time schedule of the project, but also drove a loss of passion for the project; as being stagnant for so long greatly reduced motivation.

It was the eventual realisation of the lead researcher that these setbacks may just be academic versions of real life setbacks within the business world and it was a chance to learn how to overcome an obstacle to chase the goals of the project. For example, a supplier may need a specific part for the product/solution and this will take time to source from a manufacturer. However, after being "kept on the bench" for several months, it was imperative for the true development of the project and the pursuit of the Masters qualification itself that the lead researcher drive the momentum of the project higher in order to achieve the desired results on time.

Lastly, a barrier of the project was presented as more information was gathered and different opportunities were presented. The change in market need from our initial perceptions and assumptions altered the very course of the project and it was within this 
change that the barriers were presented. From our initial assumptions that our device was a winner and could do everything an accredited lab could do, to the realisation that we could not provide an answer to everything within this industry, despite our high level of confidence in the potential of the device. We then needed to re-examine our proposed solution to the market need and assess whether our device was truly capable of filling the need. When the interview with ASDTL was conducted, it became apparent as to where exactly the device could be of strong benefit to this industry, coupled with the opportunity to work with them to bring the device to fruition truly overcame this barrier.

\section{Reflection on the Development of Our Assumptions:}

Our assumptions for the device and its relevance to providing a solution for the industry of testing athletes for signs of drug abuse and doping in sport pivoted and grew as the project developed.

Initially we assumed that all samples that were collected were only sent away to an accredited lab as a security measure to prevent cheating and have the athlete avoid prosecution whilst winning an event not fully on their own merit. This assumption proved to be only partially true, as the accredited labs are set up to be a lot more scrupulous than a typical clinical laboratory when it comes to detecting substances.

Our next assumption surrounded the need within the industry for a faster, more efficient and cost-effective device to test their samples also proved only partially true. Where we had envisioned the device taking over the whole process initially, it was only through our gathering of data and talking directly to the stakeholders involved that we discovered we simply could not compete with some of the technologies being used currently. This is partially due to the technical capability of the device, but also due to the legislative requirements set in place by WADA, and the current global framework. For example, mass spectrometry and gas and liquid chromatography are all utilised technologies at WADA accredited labs. If a new substance becomes apparent, it is easier for WADA to direct the accredited labs to look for a specific variation in the results or implement a new element to the tests that detects the new substance, as opposed to implementing an aptamer based technology where a specific aptamer would have to be developed and then transported to all of the accredited labs. 
Furthermore, we assumed at the start of this project that if we could get our device into the sample collectors' hands, we could deliver results onsite and circumvent the lab stage altogether. This assumption developed throughout the project and lead us to the end goal as more information was gathered from our research participants. What we hadn't considered with this assumption was the practicality of the sample collectors delivering the test result onsite. For example, if an athlete is using anabolic steroids - a drug known to increase testosterone and produce "roid-rage" - the sample collector could be put in jeopardy when delivering the bad news to the athlete. The other factor resides in the fact that they are not police so cannot legally arrest, subdue or inspect the belongings of an athlete, irrespective of whether they are trying to build a case. This changed our assumption on the need for the product and its use within the industry, which lead us to the idea that the device could be used within the accredited labs to promote a cost saving for them and only put certain samples forward to the more expensive and stringent tests. As we found, this avenue wasn't viable as the internal testing framework was too rigid for the initial screens and that the potential value for our device actually lay in replacing the more expensive tests such as EPO.

\section{Comparisons to Previous Research and Similar Technology within the Industry:}

Aptamers are a forever growing industry with a multitude of applications that also have new developments and innovations occurring frequently. The market has continued to see positive growth as aptamers are integrated into frameworks to promote efficiency and bring overall cost down to a more economically manageable level. RNA aptamers have proven to be of high use and value in terms of therapeutic and diagnostic work, with FDA approved aptamer drugs in development and clinical trials, leading to the overall positive growth of the market for this technology ("Aptamers Market: A Global Industry Analysis and Opportunity Assessment 2016-2026, 2017).

As we found from our interview with ASDTL, there have been previous attempts to integrate aptamers into the athlete drug testing industry. The last being in 2007, where the project lapsed due to technical and financial lapse. The main benefit of aptamers within this industry is that they offer economical scaling, time and cost efficiency and in their use instead of antibodies, they have advantages such as being more stable in higher temperatures, offer less variation between batches and can be made readily to target new 
substances. With the innovations on aptamer technology over the last 10 years, detecting substances within this industry will become more and more viable, creating a positive growth for the prospects of the technology and the industry as a whole.

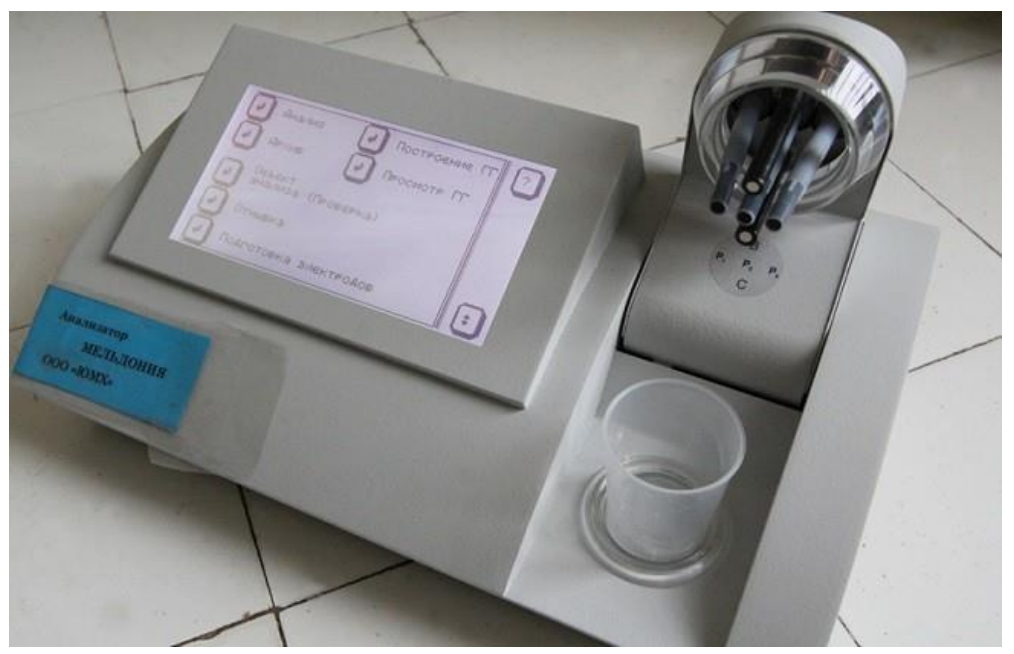

Figure 6: Prototype of an Electrochemical analyser, developed by Olga Mezentseva at Tomsk Polytechnic University. Pilot Device for Rapid Meldonium Testing - tpu.ru, 2016

The main up-and-coming competition for our device within this industry is an electrochemical analyser that has been developed out of Tomsk Polytechnic University in Russia. It is in its prototypical stage and currently has only been shown to test successfully for Meldonium, a drug commercially used to expand blood vessels for those with high blood pressure. It was banned by WADA in 2016 for it allowed athletes to potentially use it to help deliver nutrients around their body faster, aiding in recovery and performance. The main way in which Meldonium is detected at WADA accredited labs is through their mass spectrometry and gas chromatography screenings. The electrochemical technique has been used to identify many organic substances and their metabolites previously, meaning that in order for this device to adapt to detect a new or different substance, a change of electrodes would be required. The reagents required are readily available and inexpensive, and the electrodes used are a standard fit for most voltmeters. This device is expected to enter the market at an approximate cost of $\$ 2500$ NZD. It is a formidable device with a lot of promise and potential, however, as we found from our interview with ASDTL it would be hard to compete and elicit a change from the mass spectrometry techniques which WADA accredited labs have very well integrated. In the instance that this electrochemical detection device could detect EPO, we would need to promote the cost of our device, as well as the running and maintenance costs in comparison to this device. Overall, the winning device in 
this industry will be the candidate that can promote the best sustained competitive advantage.

As the athlete drug testing industry requires a lot of evidence, credibility and trust to even enter and the fact that aptamer technology is reasonably new, there is not a lot of similar technologies to compare our proposed device to within this industry. However, we can offer a competitive advantage with technologies that already exist within this industry and it will be these technologies that we endeavour to circumvent. This will create a large cost saving and promote a more efficient means to detecting certain substances which usually cost a lot more and drive up the price of athlete substance testing if further examination of a sample is required.

\section{Implications for the Device:}

The Rapid Athlete Testing project sought to explore the ability and commercial viability for Auramer Bio's aptamer based biosensor to test athletes for signs of drug abuse and doping in sport. The implications for this research suggest that the device could be used to test for substances in other areas, industries and markets as it can test within multiple matrices.

An implication for the research is that it serves as a case study example for other technologies or innovations that seek to enter the athlete drug testing industry. The Rapid Athlete Testing project has highlighted the numerous assumptions present in the general population, how these can be tested within the industry as well as which organisations to start with in order to properly assess the viability of their proposed product or solution.

Further implications for the applications of this project highlight the possibility of catering to many other markets; for example, the water pollutant detection industry. In areas where there have been signs of pollution, sickness or evidence of cantankerous pollutants in water such as Escherichia Coli (E.coli), then there may be a benefit to detecting these onsite so that treatment of the affected area could begin straight away. This carries over into the biohazard industry as a whole, as quick detection could be both beneficial and save lives, time and money, making the device a disruptive and profitable solution. This also has potential applications within the food technology industry, as a means to quickly analyse a sample to detect for signs of E.coli, Salmonella and Campylobacter. 
Another market opportunity could be the forensics market, for detection of samples and matching with potential suspects. Instead of collecting the sample, transporting to a lab, preparing the sample and running a gel electrophoresis to assess whether the sample matches the suspect, this could be completed onsite. The benefit of this is not only the time saving and cost effectiveness properties of this method, but also the reduction in risk that the sample could be exposed to microbial contamination that could affect the sample.

Lastly, the implications of the research project suggest that once a need within a market is identified, it is a matter of analysing the industry, developing a suitable business and development strategy, approaching the relevant stakeholders and customer base to assess their perception of the proposed product or solution and then making reasonable, logical decisions based on this information. Base assumptions may be proved wrong, causing a necessary pivot for a project, but the Rapid Athlete Testing project implies that more avenues may open once the relevant communities have been addressed. Overall, the initial idea may have no use within the targeted industry, however, other applications may present themselves and there would still be a path to market to make a project a success.

For example, Auramer Bio's biosensor can detect down to the femto level, so a potential application could be to use it for looking for contaminants in soil. But once the relevant industry is approached, and a stakeholder is involved, such as a grape grower that also makes wine, the project may take a turn and the device could be used to detect for known microbes that infect wine, producing oxygen which effectively turns it to vinegar. If they had a detection device, they could analyse and treat the wine to kill the microbes and save the batch, improving yield and therefore profits. 
VERSION 1.7

FEBRUARY 28, 2017

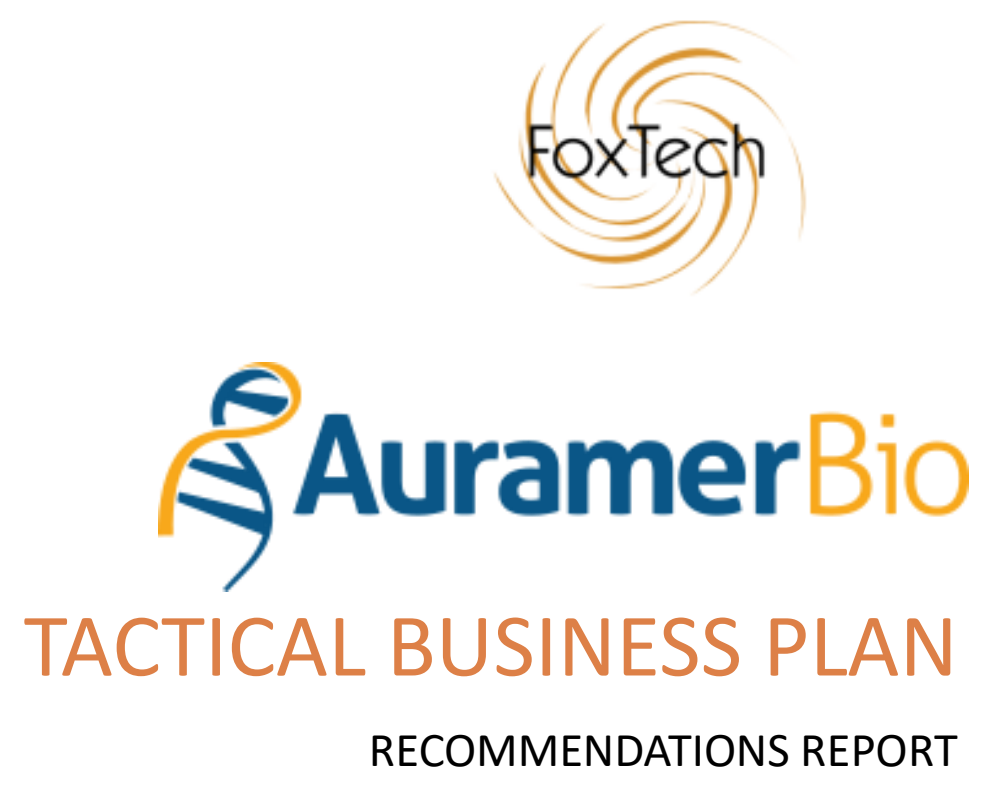

PRESENTED BY: MARK FOX

FOXTECH AND AURAMER BIO

VICTORIA UNIVERSITY OF WELLINGTON 


\section{TABLE OF CONTENTS}

BUSINESS CASE \#

\section{SECTION 1}

EXECUTIVE SUMMARY

COMPANY DESCRIPTION

$\#$
$\#$
$\#$
$\#$
$\#$
$\#$

MARKET ANALYSIS

\#

\section{SECTION 2}

ORGANISATION AND MANAGEMENT

PRODUCT LINE

MARKETING AND SALES

SECTION 3

FUNDING REQUEST

FINANCIAL PROJECTIONS

$\#$

\#

\#

\#

$\#$

\#

\#

\#

\#

\#

\# 


\section{Recommendations (Business Case):}

\section{Executive Summary:}

We at FoxTech ${ }^{\mathrm{TM}}$ are a subsidiary company of Auramer Bio, dedicated to the commercial exploration of their aptamer-based biosensor. We endeavour to isolate suitable markets through accurate industry targeting and analysis. Our core project has been the Rapid Athlete Testing project, where we explored the market need within the industry of testing athletes for signs of drug abuse and doping in sport.

Our CEO and lead researcher has a history in biotechnology and has recently expanded into the business sector of scientific industries to pursue his passions. It was towards the end of his Bachelors of Science degree he realised that his passion wasn't based at the research end of science; but more towards the business end where multiple applications could be sought and applied to help businesses, the general consumer and promote growth within the scientific industry.

It was through this passion that FoxTech ${ }^{\mathrm{TM}}$ was founded, to deliver a high-quality service and advice for companies that have stemmed from scientific innovation and breakthroughs. As a starting point, we wish to promote success for our first project before taking on further clients. We aim to thoroughly examine all ideas for potential avenues for any given technology or service by conversing directly with stakeholders, the proposed consumer base, potential investors and the end user. This is to ensure continuous growth and success for Auramer Bio within New Zealand and Australia.

For the Rapid Athlete Testing project, we had to demonstrate a thorough market analysis to find the need or gap within the market and how Auramer Bio's biosensor could provide a long-term solution, whilst forming strong relations with the clients and constant growth for the future.

To conduct our thorough market analysis for Auramer Bio, we started by learning the process for sample collection, transport, testing, specialised testing and results delivery. We analysed this for ways the athlete drug testing system could be completed more efficiently, 
which lead us through our initial assumptions. Following a thorough examination of similar technologies and how other biotechnology companies brought products to market, as well as an investigation into our potential competitors, we were ready to begin sourcing and contacting relevant industry experts for information. Following this, we were able to formally reassess our assumptions, our timeline and create a path to market for this product once a viable prototype is released.

With a FoxTech ${ }^{\mathrm{TM}}$ solution, we are confident Auramer Bio will be successful in this target market and their success will spread into other avenues. For the foreseeable future, we aim to bring this project to fruition whilst exploring more avenues and delivering superior solutions to our growing list of clients.

\section{Company Description:}

FoxTech $^{\mathrm{TM}}$ is a subsidiary company of Auramer Bio for the purpose of free artistic licensing and was developed solely to explore the capability and commercial viability for their Aptamer-based biosensor to test athletes for signs of drug abuse and doping in sport. This is a smaller-sized industry as it is highly specialised. This field of drug testing is more rigid, structured and organised than most other industries and has a lot of pressure on accuracy, reliability and cost effectiveness when it comes to the technology used when testing the samples for signs of doping.

AuramerBio is a biosensor development company that specialises in small molecule sensing solutions for markets requiring laboratory quality results in real-time. Our team have developed technologies and processes over a number of years to efficiently design and optimise high-performance DNA aptamers to measure small molecule targets in solution.

The current process within our targeted industry revolves around the collection of athlete's samples, be it urine or blood. These samples are packaged into tamper-proof kits and then the samples are transported to one of 34 World Anti-Doping Agency (WADA) accredited laboratories around the world. Here in New Zealand, all of our athlete's urine samples are transported to the only accredited laboratory in Oceania, which is based in Sydney and is known as the Australian Sports Drug Testing Lab (ASDTL). As blood has a 48-hour period to conduct analysis tests before it denatures and becomes an inviable testing medium, the geographic layout of Oceania restricts blood samples being sent away to Sydney, meaning 
our athlete's blood samples are sent to an endorsed forensics lab based in Auckland. It is within this process where results can take up to 4 weeks and the average test costs around \$700NZD. The cost of testing naturally increases if more rigorous and expensive tests are required, leading to an increase of up to $\$ 500$ per sample! We believe that with the aptamer-based biosensor under development at Auramer Bio, we can satisfy a market need to reduce the cost of these more laborious and expensive tests whilst delivering results in a more timely fashion.

The WADA accredited drug testing labs currently have a solid framework in terms of screening samples, where 92 athlete's samples can be placed in a 96-well plate to be examined for indications of banned substances. This initial screening process takes a full day, and is the gauge which defines whether a sample is selected for more scrupulous testing. The more expensive and intensive tests seek to look for substances such as Erythropoietin (EPO) or testosterone that are produced naturally by the body and therefore are looking for the difference between what the body naturally produces and the pharmaceutical product. These tests are known as "confirmation tests" as through the more expensive methods of testing, a more definitive result is secured to determine whether a banned substance or its metabolite is present within a sample. It is within the more thorough testing procedures where we have discovered a market need for Auramer Bio's device.

Auramer Bio's aptamer based biosensor is a highly sensitive device that can detect substances down to the femto $\left(10^{-15}\right)$ level! Aptamer biosensors use aptamers, which are pieces of single-stranded DNA or RNA that are complementary strands to the desired target. This means they will only bind to the desired target, which ensures accuracy and credibility in terms of the results of the testing of a sample. Aptamers are able to be made quickly to meet the market need of detecting for a new substance, they are an economic and ecological option as they are cheap to produce and run and can always be produced, unlike the antibody technology in use within the current methodologies. The anti-human mouse antibodies used in EPO testing are very specific and well-studied, however they are less stable at ambient temperatures, require cooling if they are to be transported and ultimately have lower specificity and affinity than aptamers. Antibodies also have a shorter shelf life than aptamers, this means that if they are not rotated properly they can expire. There is 
potential to utilise Auramer Bio's aptamers and their biosensor within this industry to promote cost savings on their confirmation tests, which will save the accredited labs time, money and allow them to invest more into their innovative research.

Aptamers remain stable at a variety of temperatures and will therefore remain intact and be in viable condition for use after being transported. Because aptamers can withstand a range of temperatures, they can be transported much easier in different climates around the world, and consequently will be more cost effective as they don't require refrigeration in transit. Auramer Bio's biosensor runs on specialised aptamer kits which are designed to specifically detect the target substances that our clients indicate they require. These aptamer kits can be made relatively quickly for this industry to detect new drugs as they are developed or added to the WADA banned substances list.

Some of the more complex tests can take between 2 and 8 hours solely on the preparation methods, where AuramerBio's device can greatly reduce the test turnaround times and produce the results within a reasonable timeframe of approximately 20 minutes. This fast testing feature coupled with a highly sensitive detection threshold and an almost infinite range of aptamers to bind to desired targets creates a large time and cost saving benefit, as well as a robust sustained competitive advantage.

We believe that once Auramer Bio's aptamer-based biosensor has been fully developed into a working prototype, there is the potential to fulfil a need within this market that promotes an economic, ecological and efficient alternative to their current methods. The prototype will require proof of concept testing and peer revision, however, once the proper WADA accreditation is obtained for the device, there is the potential that is can be a disruptive and effective asset to this industry.

Companies we Serve in this Industry:

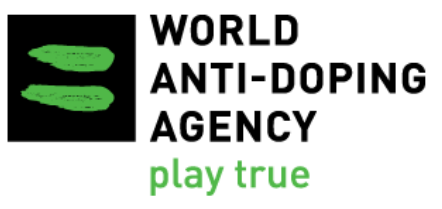

Figure 7: WADA Emblem, 2017
WADA is the main governing body for this industry and effectively decides upon all of the processes; from collecting the sample, transportation to the accredited lab and how the tests are conducted. This is highly regulated to ensure security and above all fairness in professional sport. Primarily, to enter this industry we will need to obtain the necessary 
accreditation from WADA for AuramerBio's device to be used and integrated within their framework. As the device is a novel entity, evidence of previous assessments and tests to prove its capability will need to be completed and then presented to WADA to await their decision surrounding whether or not there is enough benefit to give the device accreditation.

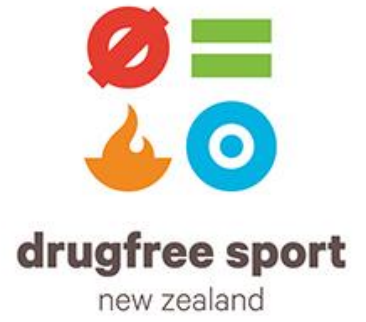

Figure 8:Drugfree Sport Emblem, 2017

Drugfree Sport NZ are the national entity for New Zealand and they manage the sample collection process, the transport of these sample either to the WADA accredited lab in Sydney, or the forensics lab in Auckland whom are endorsed to conduct tests on the blood samples. As we are based in New Zealand, Drugfree Sport NZ will be a critical partner for communications and advice for the true development of the Rapid Athlete Testing Project.

ASDTL are the only WADA accredited lab in all of Oceania and

Australian Government

Australian Sports Anti-Doping Authority

Figure 9: ASDTL Emblem, 2017 conduct the tests for signs of drug abuse and doping in athletes for Australia, New Zealand and surrounding nations. It is here that our opportunity lies for Auramer Bio's device to be tested and used within this rigid industry. During our interviews and research gathering, ASDTL could perceive the value and benefits

the device could promote and kindly offered to use the device alongside their more rigorous tests to ensure the validity of the device. Once a viable prototype is fully developed, then the testing phase can begin within the industry! The evidence gathered from the tests will have been created by ASDTL themselves and should therefore hold more weight for the eventual proposition to WADA. Overall, it will be WADA that have the final say as to whether this avenue will be viable for Auramer Bio and their device. This unexpected opportunity that was presented will bolster credibility of the device and create a firm starting point within this industry; as working with a WADA accredited lab will give light on the requirements and provide extensive feedback on any further developments that may be 
compulsory to attain WADA accreditation. ASDTL will be our key partner and foundation for the application of the device within this industry.

\section{Competitive Advantage:}

Auramer Bio have proven to generate the best in class aptamers that are further optimised within their device and its application. Currently within the athlete drug testing industry, antibodies are used as a means of detecting the differing levels of naturally produced substances, and the pharmaceutical product. The most common method of antibody testing procedures utilises the ELISA assay, which operates at the $10^{-12}$ (pico)

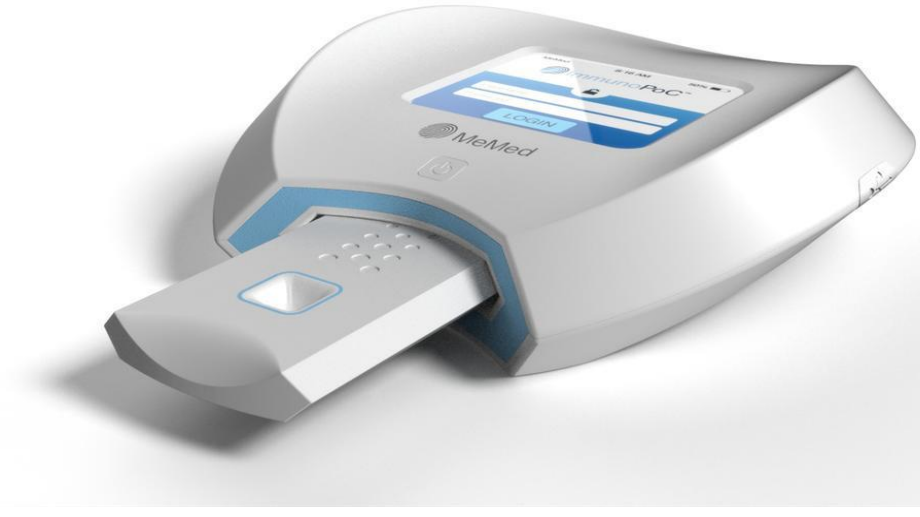

Figure 10: Proposed Prototype Design for Auramer Bios AptamerBased Biosensor, 2017 detection threshold. Auramer Bios aptamers paired with their novel device can detect substances $1000 \mathrm{X}$ smaller than the ELISA assay tests.

Another competitive advantage that Auramer Bio and their device hold is the ability for fast turnaround times for results. Disregarding the preparatory work, typical lab tests may still take at least 1-2 hours, where the device can deliver a result in approximately 20 minutes. For this market, if a more extensive test is required, the athlete has the opportunity to go the WADA accredited lab, inspect their secondary " $\mathrm{B}$ " sample to ensure no tampering has occurred and then watch the test from start to finish. With Auramer Bios device, the athlete and the lab technicians wouldn't need to spend a long amount of time conducting the tests and awaiting the results as the device can have the aptamer kit installed in front of the athlete, the sample dropped into the sample reservoir and the result will be delivered much faster than the current method. This competitive advantage will save the lab both time and money, whilst providing a much better experience for the athlete.

Furthermore, we can bring extensive value to our client base. The average athlete drug test costs $\sim$ \$700NZD, with an extra \$500NZD for any further testing required. This is due to the amount of time it takes to conduct the tests, the reagents and materials used for the tests 
and the methods used. The current machines used can cost approximately \$450000AUSD, whereas Auramer Bios device will only be a fraction of that and the required aptamer kits will be more economic expendable resources than the current methods implored by the target market.

Lastly, the company structure bolsters expert personnel in a range of disciplines, each dedicated to the success of Auramer Bio and their device. FoxTech is a side-branch company that has isolated contacts within the athlete drug testing industry, found a suitable starting point for the device and effectively has navigated a path to market through the use of the contacts collected. It is our vision to deliver a high-quality product that promotes a competitive position within the market.

\section{Market Analysis:}

\section{Industry Size and Outlook:}

The athlete drug testing industry has seen constant growth over the last 40 years. It is a dynamic industry that is a constant adaption to the behaviours of athletes trying to get a competitive edge whilst avoiding detection of drug abuse or doping. Novel performance enhancing drugs are created at an ever-growing rate and it is the responsibility of this industry to create tests to detect the potential use of these drugs. This industry is spread around the world and is managed by one governing body - WADA. There are 34 WADA accredited laboratories that conduct the tests and produce the results for the national government bodies that regulate the prosecution if infringements. This industry is highly regulated; therefore, our opportunity lies in providing sufficient evidence to WADA that the device can be well implemented within every WADA accredited lab whilst being economic and ecological. It is a good starting point as fewer, closer, longer term collaborations instead of an abundance of short term deals, have deeper and fewer deals that are broader in scope. This would lead to more sharing of information, greater joint learning, and more productive investments. Our device has the potential to be a profitable and disruptive asset within this industry.

\section{Information about Target Market:}

Our target market aims to start based out of New Zealand, working with Drugfree Sport NZ and ASDTL to gain credibility on the device and test its applications on a smaller "proving ground." The New Zealand and Australian athlete drug testing market for out-of competition tests runs approximately 5000 samples per year. This averages out to approximately 14 sample tests a day, however some of these tests are then selected for further testing for harder-to-detect drugs. This is a small starting point, but is not too small 
as it gives the opportunity for Auramer Bios novel aptamer-based biosensor to test samples in the target market to prove its effectiveness and efficacy. The key point to starting within the smaller market is to assess the device in industry as it is a prototype and make any adjustments or developments as required. In order for the device to be incorporated, it must be either endorsed or accredited by WADA, which will only happen once sufficient evidence of its capability is presented.

Currently, our potential customer base utilise well-researched and trusted but much more expensive technologies to achieve their test results. The machines for these tests are very expensive, and within the Australian and New Zealand athlete drug testing market, they currently do not conduct enough tests to make them self-sufficient or economically viable without government aid. Our target market has a critical need to conduct these tests at a more economically viable price in order to be a cost-effective laboratory that also remains competitive within the global market. We will primarily work with ASDTL as they are the only WADA accredited lab in Oceania, and they are based in Sydney, Australia. Given that they test athletes both in and out-of competition, there will be cyclical trends that affect the purchasing and use of the aptamer kits once it is WADA endorsed or accredited. In terms of larger events such as the Olympics, we would anticipate a rise in demand for the device and the aptamer kits as more tests are conducted and are much more stringent, which is where we have a competitive advantage due to the sensitivity of the device.

\section{Size of the primary Target Market:}

ASDTL and Drugfree Sport NZ work together to collect the samples, transport and test several thousand samples per year. The antibody technology which is our primary competition and what we aim to circumvent for our customer base through proven cost effectiveness and efficiency. This market is set to grow with the onset of more and more prohibited substances being added to the WADA banned substances list, and the rise in the general population also producing higher calibre athletes, of whom eventually qualify for the level of sport where drug testing is common practice. Coupling these factors with the surge in advancements from the medical field, these athletes show a trend of endeavouring to beat the system, meaning the size of even the primary target market is set to escalate over the foreseeable future. 


\section{Market Share:}

If Auramer Bio's device can be used to circumvent the more complex tests, then unless an even more competitive and hugely more sensitive technology comes along, then for our primary target market there is a substantial market share. This is circumstantial based on whether WADA would integrate the device into the framework and whether the labs would still keep their machines as another back up form of confirmation testing. Within the industry, we are starting small with our prototype, operating out of one of 34 WADA accredited labs, making up for an approximately $3 \%$ market share for complex tests within this industry. If WADA does approve the device and endorses its use for complex tests within the accredited labs, then there is the opportunity for growth as we expand further internationally. For research labs within the Biosensor market, there is an $11 \%$ market share (Global Endocrine Market, 2016).

\section{Competitive Analysis:}

Our primary form of competition for entering this segment of the target market and the industry as a whole is the older, more established, researched and trusted technologies already in use by the WADA accredited labs worldwide. These technologies have their strengths and weaknesses, being that they are established and well-understood technologies. However, they are larger, more expensive machines that also require expensive expendable materials to operate. They are time-consuming and require a lot of labour within the lab to formulate and conduct the tests.

On the basis that each WADA accredited lab purchases only one of these machines, that is $\$ 15 \mathrm{M}$ of assets to the industry. The suppliers of the machines and our competition would also provide to other labs, such as clinical or forensic labs, making them a multimillion-dollar provider of the machines and their required materials for operation. From this we can infer that our target market is quite important to them, however, we can only enter this market with the proper approval from WADA, meaning at this point we wouldn't be fully considered "competition."

\section{Regulatory Restrictions:}

The New Zealand and Australian legislation and laws surrounding the entire process of testing athletes for signs of drug abuse and doping in sport refers to the adoption of the 
WADA code. This code is subject to change at the discretion of WADA, allowing for newer and innovative testing techniques to be applied as technology advances. The regulatory restrictions for our novel biosensor entering the market will reside in how well the device performs within the tests that ASDTL have offered to conduct and record on our behalf, and whether WADA will perceive enough value to integrate the device into the established framework instead of the current methods. We will comply to these restrictions by proving the effectiveness of the device and presenting the results to WADA who will in turn make the final decision.

\section{Organisation and Management:}

FoxTech ${ }^{\mathrm{TM}}$ is a subsidiary company of Auramer Bio, formed to explore the commercial viability to use Auramer Bio's aptamer based biosensor in the industry of testing athletes for signs of drug abuse and doping in sport. The proposed industry is well-structured and operates as independent entities whilst being governed by a main body. It is not a massive industry and therefore is a good avenue to explore to isolate a starting point for the biosensor, proving its applications within industrial frameworks. FoxTech ${ }^{\mathrm{TM}}$ is structured to work on the sound advice of the experts from Auramer Bio, whilst independently exploring this industry to relay the information back to Auramer Bio regarding the development of the project through direct conversation with Auramer Bios CEO.

Mark has a background in Biotechnology, specialising in cellular
processes with a passion for Intellectual Property law. Mark also
has a long history in retail, where he developed skills in people
management, extensive customer service and representation, as
well as a critical ability to think for the customer to guarantee the
total solution for their needs. Mark also adds the ability to build
solid relationships with clients, colleagues and business partners.
These inter-personal skills will aid FoxTech in networking and future
projects. The Team Management profile categorises Mark as a
Concluder-Producer, revealing a practical skill of organisation and a
systematic approach to all projects. This also means that Mark likes
to reach conclusions on all tasks in an efficient and effective
manner and will consider all pros and cons when weighing out a
decision. Mark will like to impose systems early on and judge
progress based on these deadlines, ensuring projects for FoxTech
are conducted in a timely and efficient manner.




\section{Auramer Bio:}

Auramer Bio is the main parent company involved with the Rapid Athlete Testing project and owner of the technology and IP surrounding the aptamer-based biosensor. They are the instigators for the project and have provided a wealth of both scientific and business knowledge to aid in the pioneering portion of the exploration of the industry for the project.

Auramer Bio has a firm business plan for their own avenues and a sound business structure with a team of expert personnel, the company structure is laid out as such:

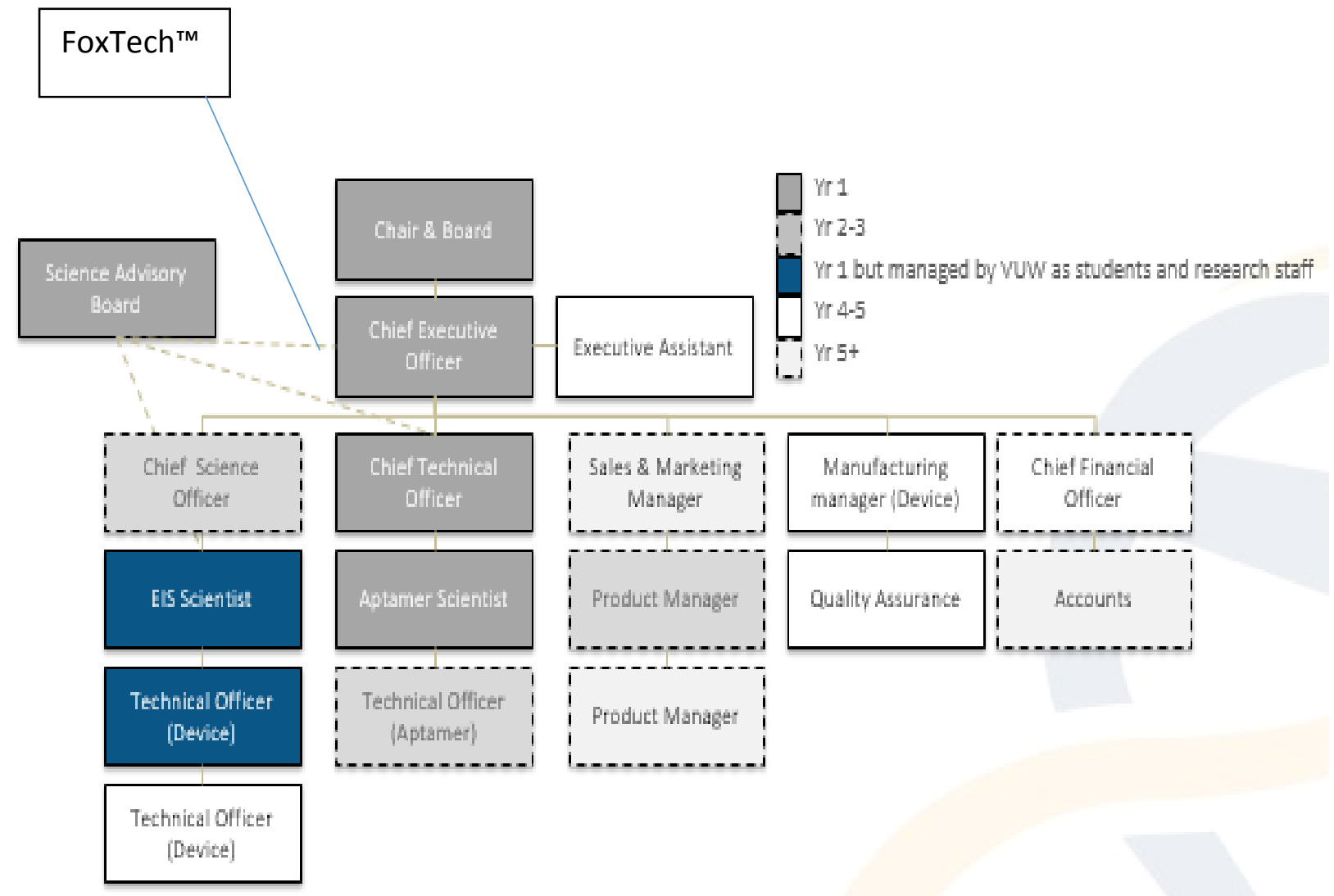

Fiqure 11: Company Structure

The company has been laid out to ensure maximum output of sound scientific and business advice to discover further opportunities for the device and its applications as well as to certify the success of the company for the foreseeable future. 


\section{Product Line:}

Auramer Bio has under development, an aptamer based biosensor which is currently in its lab component state but is being developed into a commercially suitable prototype. This device has many applications within the sensory market, from clinical applications to potential environmental sensory systems, research labs, point of care facilities and home diagnostics. The biosensor when coupled with the required aptamer kits can be 1000X more sensitive than the industry standard, promoting to the customer base a highly accurate method for testing of desired targets. The device also has a fast turnaround time for results, meaning that both the customer base and their affected clients can enjoy receiving a result within a reasonable timeframe of approximately 20 minutes, whereas the technologies currently employed within our target industry take a full day to run. Furthermore, the required aptamer kits that are made specifically for a desired substance can be made very quickly and transported easily as they remain intact at a range of temperatures and $\mathrm{pH}$ levels. Lastly, the antibodies used in our main competitor's technology have a finite life, ranging from a few weeks to a few years depending on the strain. (Jayasena, 1999). This provides a strong value proposition to our customer base, as they are then able to make a saving on the transport cost of ordering the aptamer kits as they need not be refrigerated

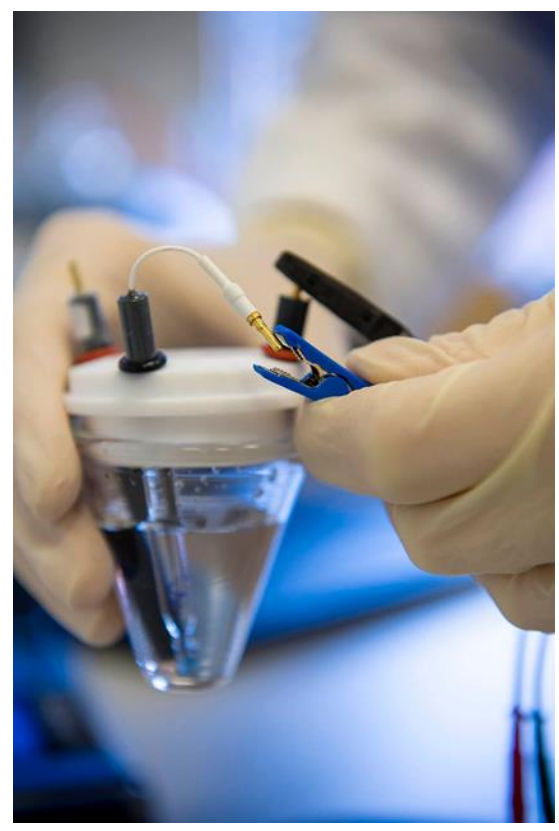

Figure 12: Lab Component Stage of the Device, Courtesy of Auramer Bio, 2017 for delivery and they also need not worry about rotating stock or managing expiry checks. This will save our customer base time, effort and promotes a cost saving that could then be turned to profit.

The product development life cycle for the device allows us to plan for stages of future development, when and where we can market our product and the eventuality where technology will overtake and innovate, leading to a more robust technology taking its place, this is known as planned obsolescence and is a key idea to keep in mind given the current rate of development of technology. Currently, the device is in its lab component stage, leading into early prototype development. Alongside this, Auramer Bio is developing a catalogue of aptamer kits ready for industry use, which will expand as over time through 
research and development projects and client demand. From this starting point, we have allowed a 5-year plan for the device to enter and be used within the athlete drug testing industry.

\section{Market Entry and Product Life Cycle:}

The first year will be spent continuing research and development of the device to make it into a commercially viable prototype. In the last 3 projected months of research and development, we begin contacting our research participants to ensure they are still interested in aiding our data collection and eventual path to market. The following $12-18$ months will then be spent in pre-entry market testing, where our device will be used by ASDTL and data can be collected to provide evidence to WADA of the devices capability. Following this, there are WADA regulation checks which should take approximately 12 months, after which we learn whether the device is endorsed or accredited by WADA for use within the accredited labs of our target industry. After a maximum of 42 months, the device could be fully integrated into the industry and therefore be ready for worldwide sales to the other accredited labs. We envision each lab will need several of the devices and a multitude of aptamer kits ready to complete the more rigorous testing. In this time, we will endeavour to keep up with the technology advances to remain relevant and have a sustained competitive advantage. Assuming no miracle breakthroughs in detection technologies or convincing athletes not to dope, we can visualise at least a 10 year span within this industry. 


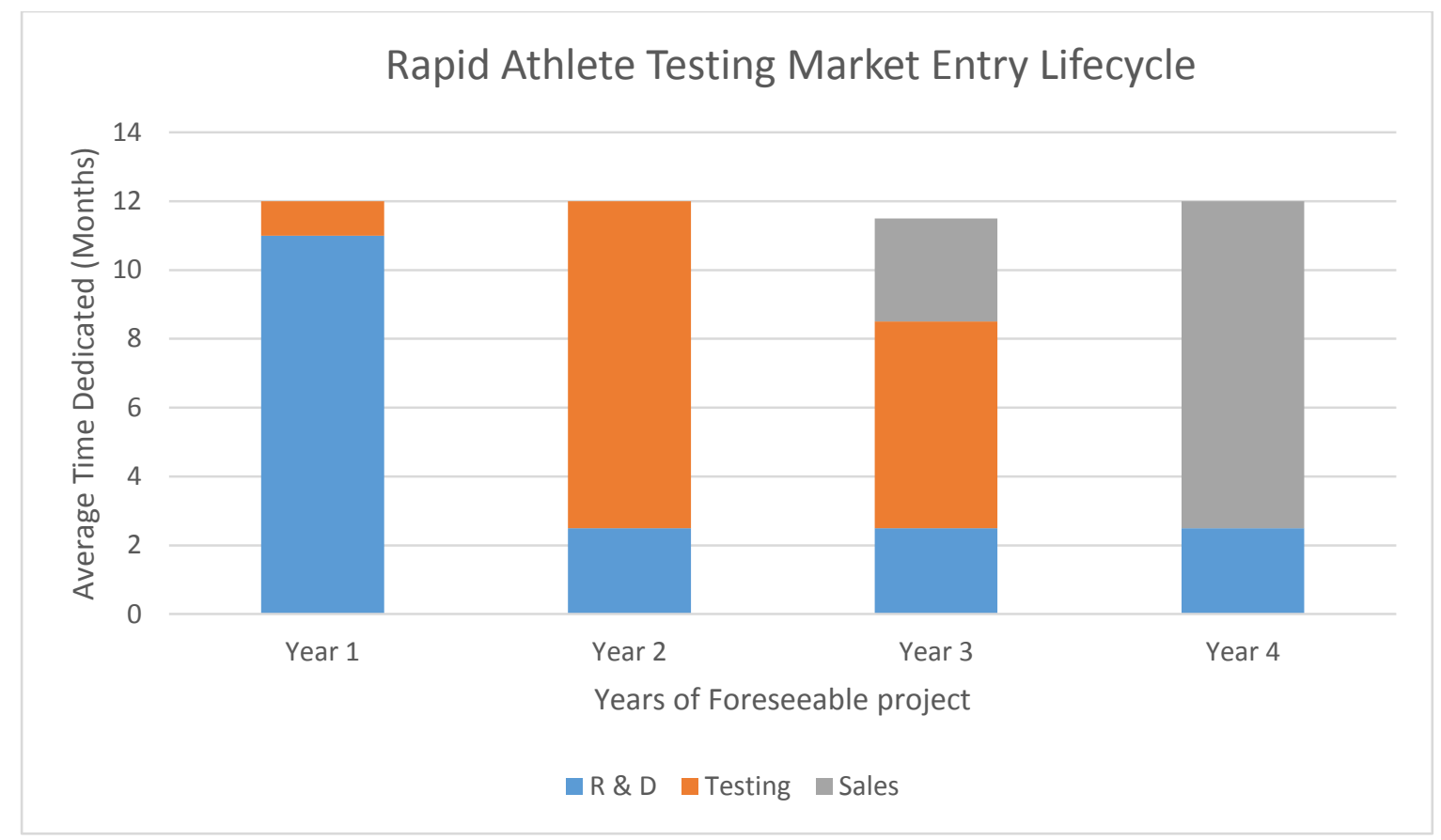

Figure 13: Market Entry Lifecycle

\section{Intellectual Property:}

FoxTech $^{\text {TM }}$ holds no intellectual property to do with the Rapid Athlete Testing project, all work has been conducted as part of a Masters course on behalf of Auramer Bio. Any intellectual property created through this project is the property of Auramer Bio, Viclink and Victoria University of Wellington. Auramer Bio has filed within New Zealand for the intellectual property of their sensory technology and their aptamer digestion methods, as well as the intellectual property filed through the United States for their aptamer technology use in detecting hormones, hormone mimics and the metabolites thereof. They also have several other patents either under examination or awaiting examination and are therefore a trade secret.

\section{Research and Development Opportunities:}

The future research and development opportunities for the proposed product line entail amassing a catalogue of aptamers for direct sale to established clients. Another key step is working the aptamer-based biosensor from its lab component stage into a viable prototype. We anticipate over the life cycle of the device that many new substances will become targets that require detection within our target industry, and therefore research and development time will go into developing new aptamers as the client indicates as well as seeking future technology opportunities to include in future versions of the device. This will 
allow us to keep the clients happy as they can have the latest aptamer sensory technology at their disposal, as well as maintain our foothold in the market for a sustained competitive advantage. Research and development will be critical to the potential continued success of the Rapid Athlete Testing project as innovation is a continual process that is necessary for maintaining a disruptive and profitable presence on the market whilst establishing good relations with both already confirmed clients and prospective new clientele.

\section{Marketing and Sales:}

\section{Overall Marketing Strategy:}

The market penetration strategy has been outlined and is simply working with a WADA accredited lab, ASDTL, in order to test and peer review the capability of the device in our target market to observe and record its effectiveness. This evidence will then be collected and presented to WADA to ascertain whether they support the device and would be willing to give funding for further efficacy testing. If WADA can perceive enough value in the device to provide funding and push for further regulatory tests, then it is likely they may endorse the device or give it the proper accreditation, which will then allow entry into this market and industry.

The growth strategy for the Rapid Athlete Testing project once the potential WADA endorsement or accreditation is obtained, is a horizontal strategy where we can deliver the device and the aptamer kits across all of the WADA accredited labs worldwide. If the devices value is also perceived by WADA for its potential use in the sample collectors' hands, then a vertical strategy can also relatively easily be implemented to distribute our product line at different points of the distribution chain.

Our channels of distribution strategy involve several tiers for effective execution. Once we have a WADA accredited commercially viable biosensor device and can measure demand, we will need to source components for the device. For items such as the electrodes, we will need to source an original equipment manufacturer (OEM) and organise an agreement so that we can obtain these for the best price possible to develop into the biosensor and operate as a value-added reseller of the technological components. Next, we would need to 
operate an internal sales force to gauge interest from the other WADA accredited labs to gauge their interest on the device. Through the WADA accreditation process they should be well aware of the potential value the device can deliver, including the time and cost savings the device promotes. The device and the aptamer kits themselves don't require special temperature controlled transport, so can be couriered directly to clients, promoting a competitive advantage to current technologies employed by the target market as the client can save money on the cost of ordering our aptamer kits.

Our communication strategy we could utilise involves a plethora of viable techniques including: promotions, advertising, personal selling, brochures and public relations. For the Rapid Athlete Testing project, our main avenue to contacting the WADA accredited labs would be through the WADA website. Here we can see all of the labs by location, have instant email and phone contact to their directors, and will be able to quickly and efficiently organise time to discuss the potential business applications we could fulfil for each accredited lab as their needs may be different. This direct approach to personal selling and public relations with the accredited labs should create a good relationship and degree of trust, boosting further business with them and their facilities.

Given that there are 34 WADA accredited labs worldwide, each with varying amounts of samples tested each year, the degree of sales to each lab will vary. Some may need only 3-4 devices whereas other labs may require at least 15 , the needs of these clients will fluctuate from lab to lab, but whilst the device does deliver a good degree of profit, the main revenue stream will come from the continued sales of the required aptamer kits, similar to purchasing ink for a printer - the big money isn't in the machine itself, but in its consumables. Finally, we will also require a market access partner or distributor at alongside the project - we are a tech development company and use a partnering model to get products and services to market.

The sales strategy itself will need to cover several milestones to be viable for Auramer Bio, so after the WADA accreditation or endorsement procedures are completed it is necessary to gain sufficient contracts from our prospective clients in the accredited labs. As more contracts are negotiated and finalised, revenue streams can develop and the cost of research and development can be mitigated for previous work done on the Rapid Athlete Testing project and for potential future projects. 


\section{Funding Request:}

In order for the Rapid Athlete Testing project to succeed we need to seek funding to aid in the true development of the product and the foreseeable future of the project. For the next year, we endeavour to develop the lab component device into a minimum viable product or prototype that we can bring to our potential clients and show the effectiveness of the biosensor within this industry. In order to comfortably achieve this goal, we will require approximately $\$ 500,000$ - $\$ 800,000$ to develop the prototype. This will cover the costs of acquiring the components and the perpetual development and testing to ensure that our high level of accuracy is maintained as we venture from a lab product to a commercial entity product.

For the future aspirations and goals of the Rapid Athlete Testing project, we have a market penetration strategy that involves the opportunity of working alongside the only WADA accredited lab in Oceania, ASDTL. For this area of the project, we will work with and license out our technology for the use in their labs to test athletes for signs of drug abuse and doping in sport. This is a fantastic opportunity to test the potential applications of the device from within the target market, and also will create evidence of the abilities of the device for the entirety of the athlete drug testing industry. We envision this stage will take between 12 and 18 months. For this critical phase of product, the development and pre-market testing, we will need somewhere in the vicinity of 1 million dollars.

Furthermore, for the marketing, distribution and early market entry stage of the project, we will need a further 1 million dollars to secure our place on the market and begin to operate with our manufacturer and market access partner or distributor. This is the final phase of the project's entry to market, from here we anticipate growth within the biosensor industry as a whole which will increase other potential avenues and therefore our revenue streams. As there is the potential for both a horizontal and vertical integration strategy within this target industry, there is a high chance of reaching more clients and therefore creating a greater opportunity for a successful return on investments. 


\section{Financial Projections:}

FoxTech $^{\mathrm{TM}}$ is a start-up subsidiary company of Auramer Bio, dedicated to exploring the commercial viability of their aptamer based biosensor within the athlete drug testing industry. As a start-up, we don't have an established financial for creditors or potential investors to inspect, so we must provide prospective financial data on what we expect this project to achieve over the next five years.

However, the biggest complication as a start-up company that is branched off from another start-up company, where the main piece of technology for market entry hasn't been developed; is knowing the proposed pricing schemes for the device and the required aptamer kits. Overall within our funding request we have calculated an approximate 2.8million-dollar fund will carry this device into the international market. According to the WADA anti-doping testing figures - 2014, on a global scale, approximately 300,000 tests are conducted worldwide every year. If the device were used within this testing framework and we disregard the cost of the device itself and any licensing costs, we could hypothetically charge an average of $\$ 5$ per test. The industry is conducting around 822 tests per day, that's \$4110 per day. That means that within 22 months, the initial investment of funding to get the project off the ground will be reimbursed, and further profit from there can go into more research and development, and/or the exploration of other projects. 


\section{Conclusion:}

The Rapid Athlete Testing project was formed from the Masters of Innovation and Commercialisation course run by Victoria University of Wellington in partnership with Auramer Bio. The research conducted revealed the structure of the industry, the number of parties involved and the overall process being utilised by these parties. The Rapid Athlete Testing project also exposed how this tightly-regulated and security-conscious industry isn't hugely complex, as only a few organisations are involved from a national standpoint. There is the national drug association, like Drugfree Sport NZ, who collect the samples and organise the delivery of these samples to a WADA accredited lab. And then there's the nearest WADA accredited lab geographically that the samples will be sent to for analysis and testing. Lastly, there's WADA themselves, who govern over the entire process globally to ensure fairness within sport. The process in New Zealand involves taking a sample from an athlete, if it's urine it will go to ASDTL in Sydney, if it's blood it will go to Labtests Auckland Ltd, as New Zealand is geographically limited in serving the practical development of the Athlete Biological Passport program. This is because urine can be frozen and transported, whereas in blood, freezing the sample can destroy potentially infringing metabolites. Blood also has a 48-hour period for the sample to be analysed for signs of drug abuse and doping, so transport to ASDTL would be inviable as the sample would denature.

This research project highlighted the general perception of drug testing in sport - that the current method of urine collection is invasive, but a necessary evil to guarantee a fair game for all athletes. Urine is still the most suitable medium for the labs in terms of testing as the metabolites are better understood and remain intact in the sample for a longer period. Blood testing used to be comprehended as more invasive, but the research project found that most athletes would prefer to give a blood sample, as opposed to having a sample collector inspect their genitalia and then watch them pass a sample.

Furthermore, the research itself highlighted the need within this industry for a more effective means of facilitating the testing of more high-end, complex metabolites. This is where the project pivoted from a device to circumvent the industry, to an indicator pre- 
screening which samples to send to the accredited lab to eventually find a suitable starting point as a means for economical testing of naturally-produced metabolites and, through our generous research participants, a potential path to market. Couple with this potential path to market would come with the prestigious WADA accreditation for the device, which would not only mean that it is viable within this industry, but accurate, highly-tested and scrupulous enough to be an effective asset within any biosensor market or industry.

The research also highlighted the fact that whilst this industry has a complex role, its structure is quite simple, having only a few involved to interview and conduct research analysis. There is also never a guarantee that a company could enter this market as WADA is the governing body that decides the fate of all prospective projects. In theory, the Rapid Athlete Testing project could serve as a set of guidelines for other companies and their technologies that could potentially enter this industry, but must keep in mind that this will only occur with sufficient evidence and WADA's approval.

The most important implications of the project involve the increasing need to replace antibody technology with something more economically viable to remain competitive in a growing market. The Rapid Athlete Testing project explored the use of Auramer Bio's aptamer-based biosensor within the athlete drug testing market, though the implications of the project could extend into other research labs, clinical experiment labs or even forensics. The biosensor industry is set to increase to a 10.5 billion dollar industry by 2021, with an average compound annual growth rate of $8.5 \%$ (Research, Z.M., Global Endocrine Testing Market.., 2016).

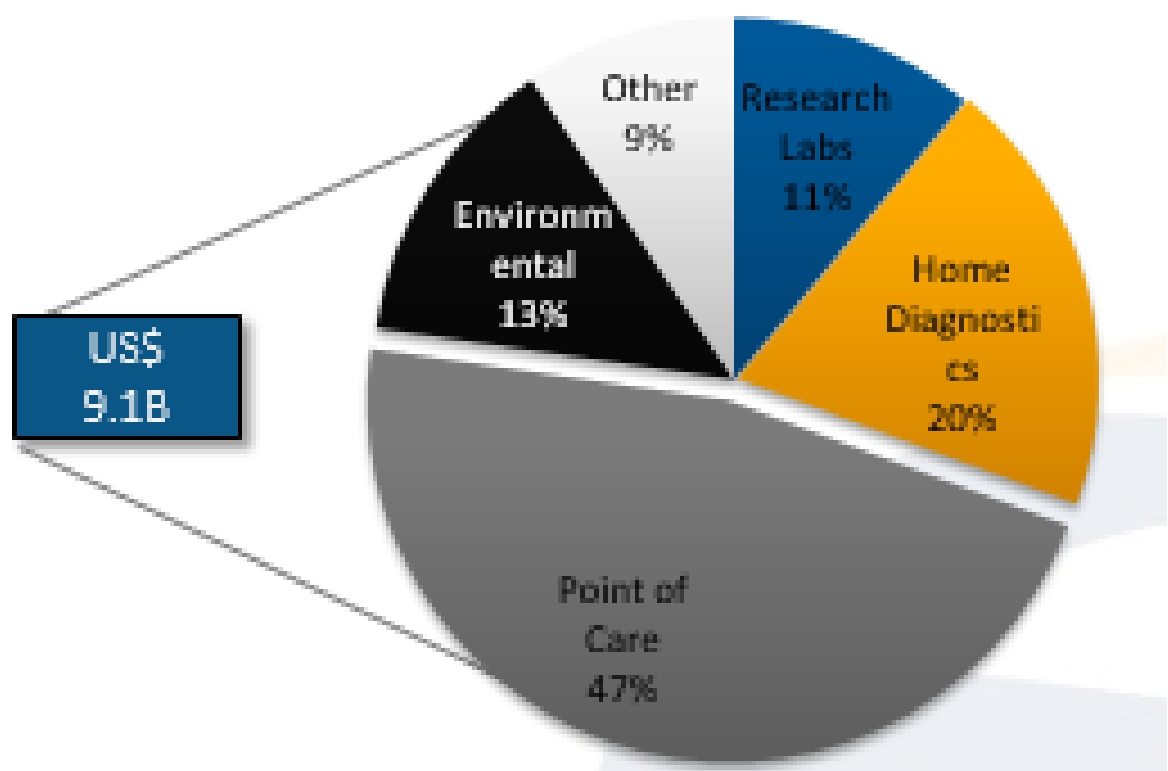

Biosensor Market 2012

Figure 14: Biosensor market 2012, courtesy of Auramer Bio 
As the biosensor market has an $11 \%$ share utilised for research labs, and the growth rate of the industry set to expand further, the implications of the research for this project suggest that by establishing this device within this industry early, we could potentially capitalise on the prospective market growth rate and become a reputable vendor in the future for expansion into multiple other fields. Auramer Bio is already exploring the potential for the largest market share within point of care, so further exploration could entail researching the commercial viability of other fields for the device, encapsulating a share of the market within each area. With New Zealand's focus on being a "clean, green country", we believe there could be a clear and suitable avenue for a starting point within the environmental portion of the biosensor market. As the device is portable, the results of testing for potential pollutants could be gathered onsite, as opposed to being sent to a lab to await analysis, making for a more efficient treatment method and a faster response to any ecological issues.

The future of the Rapid Athlete Testing project is uncertain as this project was conducted as part of a Masters research thesis within a new Masters' program. The information gathered and contained within this final report will be handed to the CEO of Auramer Bio, and a meeting set up after the submission date to discuss the key findings. All information must be kept securely for 3 years following the submission of the thesis report. The lead researcher will deliver the information to Auramer Bio and will effectively hand all future decision-making for the project over to the company. The CEO and the advisory board then have the all of the information regarding the exploration of their device within this industry to decide whether it would be a suitable and viable venture for the company. Auramer Bio can then utilise the collected contacts within this industry if they wish to pursue the proposed starting point for the device, once they have developed it from its lab component stage into a minimum viable product. Following on from this project, the lead researcher will no longer partake in the exploration of this industry on Auramer Bio's behalf, instead pursuing other passions of innovation and exploration within the alcoholic beverage market.

In summary, the Rapid Athlete Testing project started later in the course as the lead researcher made a pivot from their original project. There were obstacles to do with the University's Ethics committee, which withheld the development of the project and caused a delay in the starting point of the research. Fortunately, the industry has a simple structure, which did limit the amount of available research participants, therefore making up the lost time and they were completely more than capable of providing the required information for a proper industry analysis. The project itself did pivot and change direction a few times, as is the exploratory nature of the course, however still concluded with a positive result for the potential applications for Auramer Bio's device to be used within the desired target industry. 


\section{References:}

Abushareeda, W., Fragkaki, A., Vonaparti, A., Angelis, Y., Tsivou, M., Saad, K., Georgakopoulos, C. (2014, March). Advances in the detection of designer steroids in antidoping. Retrieved November 10, 2016, from http://www.future-

science.com/doi/abs/10.4155/bio.14.9?src=recsys

Adams, R. (2010). If you build it will they come?: Three steps to test and validate any market opportunity. Hoboken, NY: Wiley.

Blank. S, Dorf. B (2012) The Startup Owner's Manual: The Step-by-Step Guide for Building a Great Company. Pescadero, Calif: K \& S Ranch, Inc.

Charvat, J. (2003). Project management methodologies: Selecting, implementing, and supporting methodologies, and processes for projects. New York: Wiley.

Ellington, A D, Szostak, JW (1990) In Vitro Selection of RNA Molecules That Bind Specific Ligands. Retrieved July 10, 2016.

Holt, R. I., Erotokritou-Mulligan, I., \& Sönksen, P. H. (2009). The history of doping and growth hormone abuse in sport. Growth Hormone \& IGF Research, 19(4), 320-326.

Insights, F. M. (2016, April). Aptamers Market: Global Industry Analysis and Opportunity Assessment 2016-2026. Retrieved February 23, 2017, from http://www.futuremarketinsights.com/reports/aptamers-market

Jayasena SD: Aptamers: An Emerging Class of Molecules That Rival Antibodies in Diagnostics. Clinical Chemistry 1999, 45:1628-1650. 
Mazzei, F., Antiochia, R., Botrè, F., Favero, G., \& Tortolini, C. (2014, January). Affinity-based biosensors in sport medicine and doping control analysis. Retrieved November 10, 2016, from http://www.future-science.com/doi/abs/10.4155/bio.13.308

McCauley TG, Hamaguchi N, Stanton M: Aptamer-based biosensor arrays for detection and quantification of biological macromolecules. Analytical biochemistry 2003, 319:244-50.

Research, Z. M. (2016, November 23). Global Endocrine Testing Market expected to reach USD around 10.50 Billion by 2021: Zion Market Research. Retrieved October 26, 2017, from https://globenewswire.com/news-release/2016/11/23/892243/0/en/Global-EndocrineTesting-Market-expected-to-reach-USD-around-10-50-Billion-by-2021-Zion-MarketResearch.html

Ries, E. (2011). The Lean Startup: How todays Entrepreneurs use Continuous innovation to Create Radically Successful Businesses. New York: Crown Business.

Stoltenburg R, Reinemann C, Strehlitz B: SELEX-a (r)evolutionary method to generate highaffinity nucleic acid ligands. Biomolecular engineering 2007,24:381-403.

Teece, D. J. (2010). Business models, business strategy and innovation. Long range planning, 43(2), 172-194.

Tombelli, S, Minunni, M, Mascini, M, (2007, March 23). Aptamers-based assays for diagnostics, environmental and food analysis. Retrieved July 11, 2016, from https://www.ncbi.nlm.nih.gov/pubmed/17434340 
Tuerk, L., \& Gold, C. (2007, June 24). Systematic evolution of ligands by exponential enrichment: RNA ligands to bacteriophage T4 DNA polymerase. Retrieved February 23, 2017, from https://www.ncbi.nlm.nih.gov/pubmed/17434340

University, T.P. (2016, May 18) Pilot Devices for Rapid Meldonium Testing to Be Released in 2016. Retrieved June 29, 2016, from https://tpu.ru/en/about/tpu today/news/view?id=936

Yan AC, Levy M: Aptamers and aptamer targeted delivery. Rnabiology 2009, 6:316-320. 
Appendices

Appendix A: Strategyzer Business Model Canvas

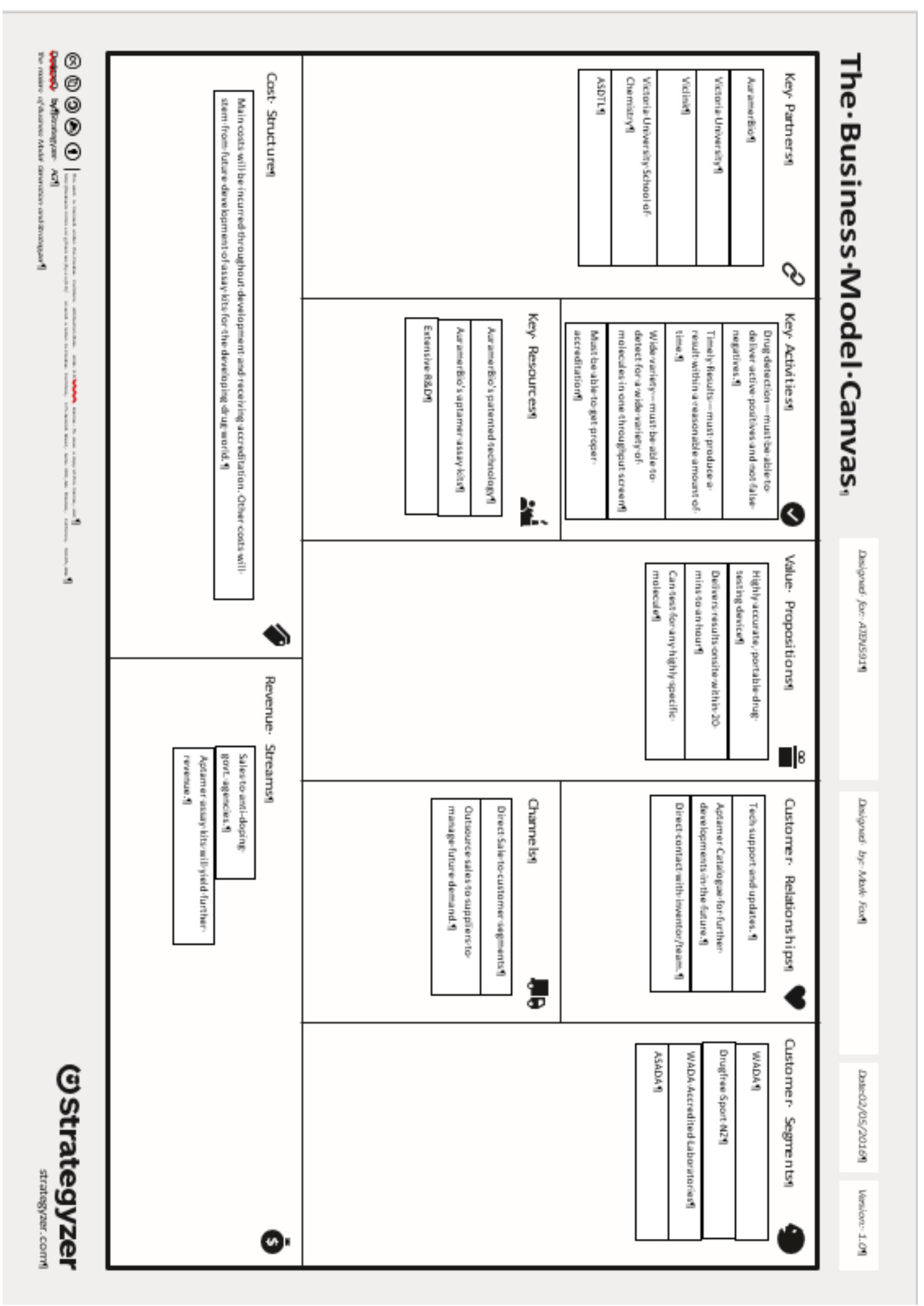


Appendix B: Lean vs. Waterfall:

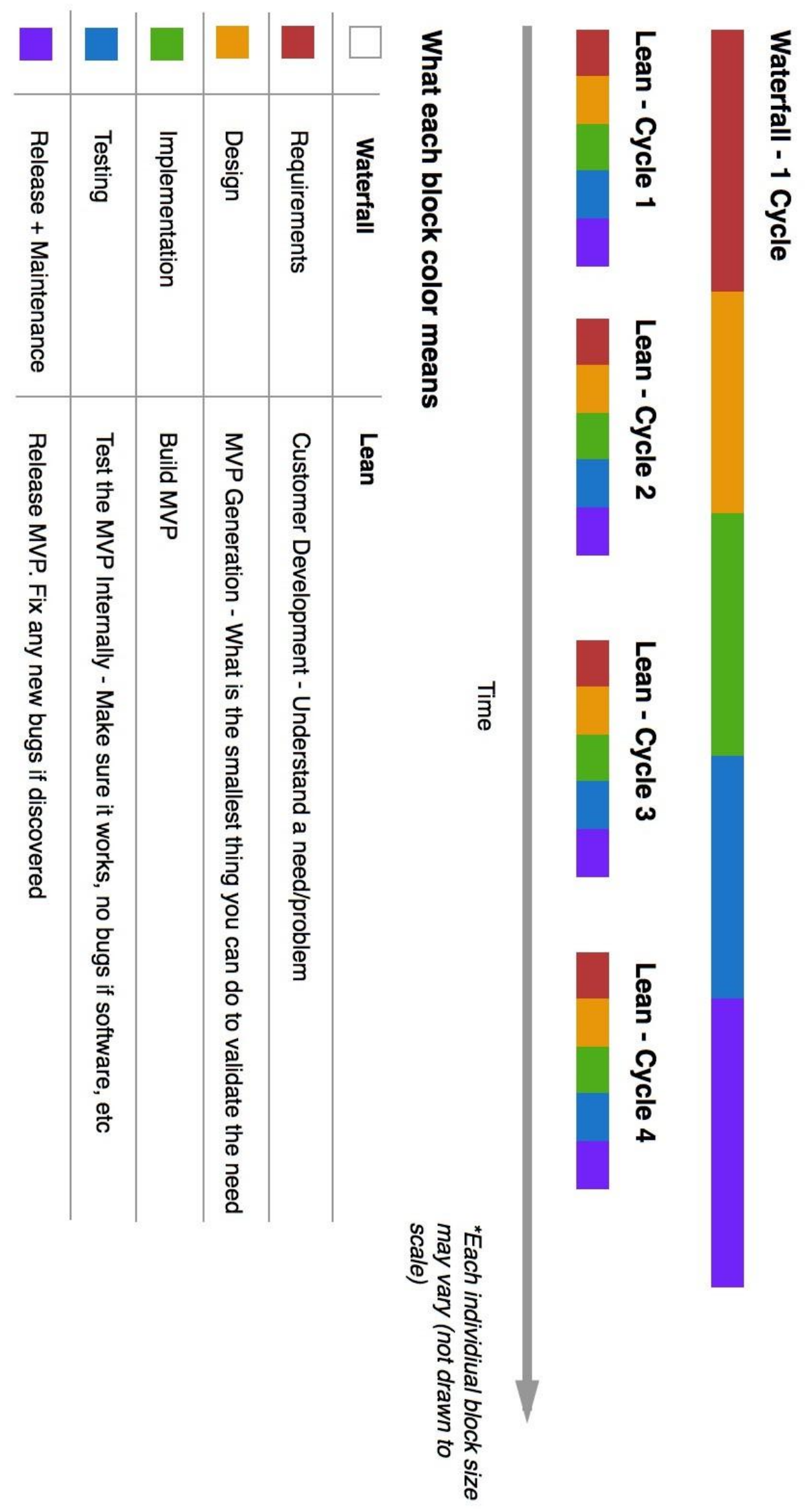


Appendix C: Example of "Cold Email”:

Dear Sir/Madam,

I am a Master's Student at Victoria University of Wellington, my course is the Master's of Innovation and Commercialisation:

http://www.victoria.ac.nz/science/study/postgraduate/spec-masters/advanced-technologyenterprise?utm source=mate shorturl\&utm medium=shorturl

For my project, I am partnered with Auramer Bio to explore the idea that their technology could be used to test athletes for signs of drug abuse and doping in sport.

In our course, a key component is to explore the commercial viability of an idea and show relevant exploration. So, whilst the idea may never come to true fruition, we must demonstrate relevant and logical decisions as the project develops. This includes talking to key people in direct and related industries to gather information.

(Stakeholder specific sentence summarizing my understanding of their involvement within the industry)

To further my research, I would really like to conduct an interview with you to learn more about your perception of the current processes and your interpretation of my proposed idea.

Thank you for your time.

Kind Regards,

Mark Fox 
Appendix D: Interview Questions:

\section{Drugfree Sport NZ:}

\section{Questions relating to existing behaviours:}

What is the current method in place for drug testing athletes?

How are these tests conducted? (ie. How are the samples taken? Etc.)

What are the current timeframes involved when a test is being conducted?

Are their limitations on what sample matrices are allowed to be tested? (Sweat, saliva, blood etc.)

How much does each test currently cost to run?

How large is your current testing pool of athletes?

In regards to the Athlete Biological passport program, what biomarkers are monitored?

How are the samples taken for the biological passport program?

Are all samples sent away to a WADA accredited laboratory or are you able to contract a local lab to run more basic tests?

\section{Questions relating to barriers to changing behaviours:}

Is there legislation in place restricting the current method of testing to its current form? (If so) What are the restrictions in place on the current methods?

According to your website, most samples in NZ go to a lab in Sydney, why is this the case? Are there any contractual limitations confining you to working with this lab in Sydney?

Would it be more beneficial to be able to test for specific biomarkers or substances on site rather than send it away for a result that can take up to four weeks?

\section{Questions relating to the proposed product/solution:}

If we could provide a handheld device that could test for a specific target in a sample and provide a result within an hour, what would be your response to this proposed idea?

What would the perceived value of such a device be?

Do you have any concerns about such a device?

Under the assumption that the proper accreditation could be gained on such a device, and clear value could be shown in its use, could there be an opportunity for its usage in drug and doping testing in sport?

If you could then take control of the sample collecting and testing using such a device, would there be a perceivable value in skipping the laboratory step?

Are there any features you can think of that could be useful in a handheld device? 


\section{WADA Accredited Laboratory}

\section{Questions relating to existing behaviours:}

In the battle against drug abuse and doping in sport, what are the key substances being abused and how are they detected?

What are the current standards set for WADA accreditation?

How many tests do you conduct on behalf of Drugfree Sport NZ and ASADA?

In the lab how much do tests cost to run?

How many samples are done in a single run?

How much does each test currently cost to run? (Including equipment, time etc.)

From my literature research it says for athletes to allow sample test results to take up to four weeks. How are samples stored during this time?

\section{Questions relating to barriers to changing behaviours:}

Is there legislation in place restricting the current method of testing to its current form?

What is your preferred matrix?

Have you recently obtained new testing equipment that may create a hurdle to changing the way tests are conducted?

\section{Questions relating to the proposed product/solution:}

If we could provide a handheld device that could test for a specific target in a sample and provide a result within an hour, what would be your response to this proposed idea?

What would the perceived value of such a device be?

Under the assumption that the proper accreditation could be gained on such a device, and clear value could be shown in its use, could there be an opportunity for its usage in drug and doping testing in sport?

Would you be willing to use this instead of the current lab testing set up?

Are there any features you can think of that could be useful in a handheld device? 


\section{WADA}

Your website lists that there are 34 WADA accredited labs worldwide, why are there so few? In the battle against drug abuse and doping in sport, what are the most prominent substances being abused?

How are new drugs selected and tested before they are put on the prohibited register? How has drug abuse and doping in sport evolved over time for WADA?

When it comes to the testing portion of WADA's involvement in drug abuse and doping in sport, are there any contractual obligations that make labs test samples in a particular manner?

(If so) what are these obligations?

Are there much more stringent tests when it comes to big events?

(If so) How much more rigorous does the testing become?

When tests are conducted for big events, are they often prioritised to get results faster than the standard four weeks waiting time?

Could it be possible to have a "Handheld Lab Indicator" device gain WADA accreditation?

What features would it have to prove to gain accreditation?

Could WADA perceive value in such a device? 
Appendix E: Survey Questions for Coaches/Athletes:

\section{Questions for coaches/athletes}

What is your experience with drug testing in sport?

How have you found the current process?

What works well?

What could be improved in your opinion?

Would it be beneficial as athlete or coach to have a portable, handheld device that could test for any substance?

If we could provide a handheld device that could test for a specific target in a sample and provide a result within an hour, what would be your response to this proposed idea?

What would the perceived value of such a device be?

Do you have any concerns about such a device?

Are there any features you can think of that could be useful in a handheld device? 